\title{
Polar Thermoplastics with Tunable Physical Properties Enabled by the Stereoregular Copolymerization of Vinyl Ethers
}

\author{
Aaron J. Teator, Travis P. Varner, Paige E. Jacky, Karolina A. Sheyko, and Frank A. Leibfarth* \\ Department of Chemistry, University of North Carolina Chapel Hill, Chapel Hill, NC 27599 (USA)
}

\begin{abstract}
A series of isotactic, semicrystalline vinyl ether copolymers (up to $94 \%$ meso diads) were synthesized using a chiral BINOL-based phosphoric acid in combination with a titanium Lewis acid. This stereoselective cationic polymerization enabled the systematic tuning of both glass-transition $\left(T_{\mathrm{g}}\right)$ and melting temperature $\left(T_{\mathrm{m}}\right)$ in copolymers derived from alkyl vinyl ethers (i.e., ethyl, butyl, isobutyl). Additionally, a vinyl ether comonomer bearing an acyl-protected alcohol was utilized as a platform for post-functionalization. Copolymers containing the masked alcohols were shown to undergo facile deprotection and subsequent coupling with a desired acid chloride. Collectively, these results highlight the diverse material properties and expanded chemical space accessible through stereoselective cationic polymerization mediated by a chiral anion.
\end{abstract}

Vinyl ethers represent an abundant and underutilized chemical feedstock for the production of high-value polymers. The range of commercially available vinyl ether monomers enables the preparation of materials that offer a diverse array of functionality. The rich history of methodology development for cationic polymerization has resulted in multiple complementary approaches for the homo- and copolymerization of vinyl ethers. ${ }^{1-6}$ Poly(vinyl ether)s (PVEs), however, typically display low glass transition temperatures $\left(T_{\mathrm{g}}<-2 \mathrm{O}{ }^{\circ} \mathrm{C}\right)$ and are amorphous viscoelastic fluids at room temperature. ${ }^{7}$ Thus, the majority of these polymers exhibit nearly identical thermomechanical properties at common operating temperatures that are independent of side chain identity. ${ }^{8}$

One frequently utilized strategy for tuning material properties is copolymerization, whereby two or more constituent monomers are combined into a single copolymer in order to achieve the synergistic combination of properties represented by the individual homopolymers. ${ }^{9}$ Previous approaches towards modulating the physical properties of PVEs through copolymerization have focused on influencing either the thermomechanical or interfacial properties through the incorporation of a functional comonomer (Figure 1). For example, the incorporation of octadecyl vinyl ether (ODVE) into PVE copolymers has been investigated to impart semicrystalline properties into atactic materials driven by crystallization of the ODVE side chains. When copolymerized with isobutyl vinyl ether (iBVE), this approach yielded materials with tunable melting temperatures up to a maximum $T_{\mathrm{m}}$ of 43 ${ }^{\circ} \mathrm{C} .{ }^{10}$ Copolymerization of ODVE with the water soluble monomer methoxy ethyl vinyl ether (MOVE) resulted in materials which form hydrogels crosslinked by semicrystalline ODVE-rich phases. ${ }^{11-13}$ Similarly, Kennemur and coworkers recently copolymerized iBVE with a rigid, iso- sorbide-derived vinyl ether monomer in order to modulate the $T_{\mathrm{g}}$ of the resulting PVE copolymers over a $25{ }^{\circ} \mathrm{C}$ range. ${ }^{14}$ Aoshima and coworkers sought to influence the relative hydrophobicity of PVEs by copolymerizing a tertbutyldimethylsilyl (TBDMS)-protected hydroxyethyl vinyl ether comonomer with iBVE.15 Upon deprotection to reveal an alcohol-functional comonomer, the relative incorporation of hydrophilic comonomer enabled rational tuning of the aqueous lower critical solution temperature (LCST). These examples, and others, ${ }^{16-22}$ highlight the challenge of preparing PVE copolymers that simultaneously exhibit high-value functionality and desirable thermomechanical properties.

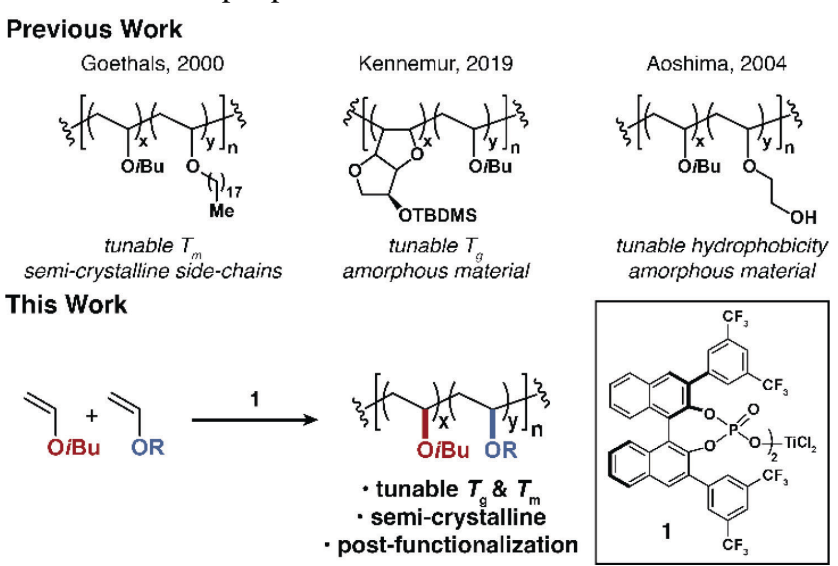

Figure 1. Stereoselective cationic polymerization using 1 enables the preparation of polar, semicrystalline thermoplastics with tunable thermal properties.

Despite the previous approaches towards the production of functional atactic PVE copolymers, the amorphous nature of these materials inherently limits the diversity of their thermomechanical properties. In contrast, polyolefins represent a high-volume and versatile class of semicrystalline thermoplastics. The incorporation of polar 


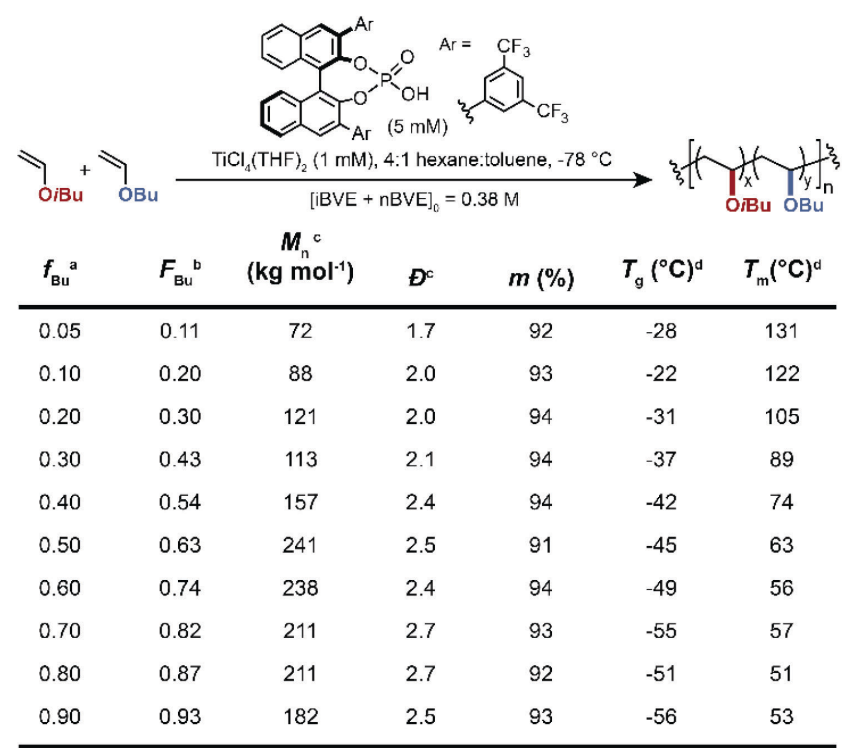

a mole fraction of $\mathrm{nBVE}$ in the monomer feed. ${ }^{\mathrm{b}}$ mole fraction of $\mathrm{nBVE}$ in copolymer determined by ${ }^{1} \mathrm{H}$ NMR integration. ${ }^{\circ} M$ indicates the number average molecular weight of the polymer. Dispersity was calculated according to $Ð=M / M$ where $M$ is the weight average molecular weight. ${ }^{\circ} T$ and $T$ obtained from second heating scan $\left(10^{\circ} \mathrm{C} / \mathrm{min}\right)$ after the thermal history was removed.

Figure 2. Reaction scheme depicting the stereoselective copolymerization of iBVE and nBVE using 1 and summary of copolymerization experiments.

\section{A}
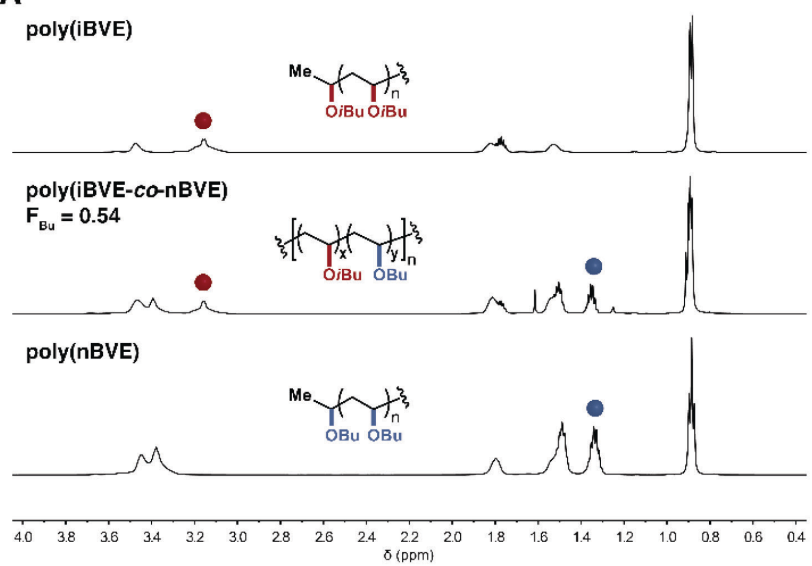

B

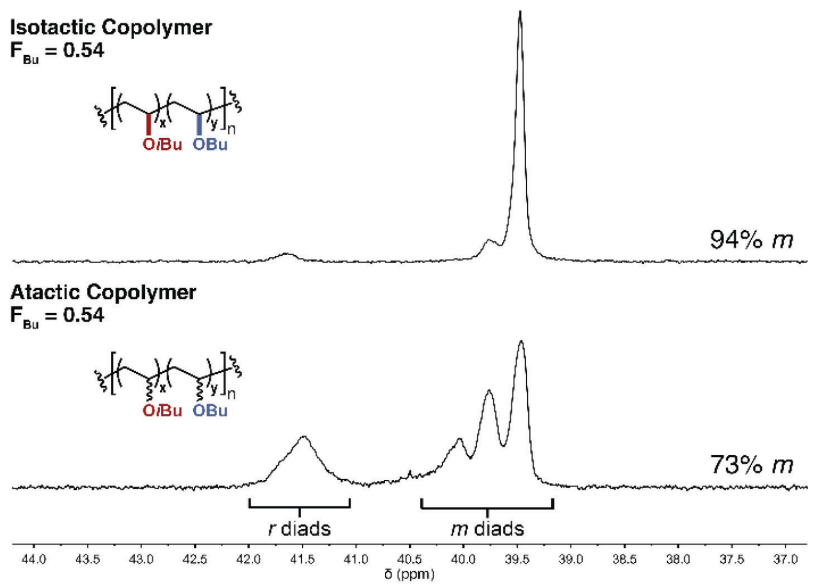

Figure 3. A) ${ }^{1} \mathrm{H}$ NMR $\left(\mathrm{CDCl}_{3}\right)$ spectra of poly(iBVE) (top), poly(iBVE-co-nBVE) (middle), and poly(nBVE) (bottom) highlighting the distinct resonances observed for iBVE (red sphere) and nBVE (blue sphere) repeat units. B) Observed differences in ${ }^{13} \mathrm{C}$ NMR $\left(\mathrm{CDCl}_{3}\right)$ resonances in an isotactic poly(iBVE-co-nBVE) made using $\mathbf{1}$ and an atactic poly(iBVEco-nBVE) made using trifluoromethanesulfonic acid. groups into polyolefins through copolymerization has been a long-standing challenge. Attempts to incorporate Lewis basic functionality into polyolefins using earlytransition metal complexes lead to catalyst poisoning and invariably limits the degree of comonomer incorporation accessible through coordination-insertion mechanisms. ${ }^{23}$ Although late transition metal catalysts have been developed to combat this limitation, ${ }^{24-27}$ the requisite activity, structural control, or stereocontrol for translation has not been demonstrated to date. The copolymerization of vinyl monomers to achieve semicrystalline thermoplastics with tunable thermomechanical and interfacial properties, therefore, remains an outstanding challenge. ${ }^{28}$

Recently, we reported a general method for the stereoselective cationic polymerization of vinyl ethers to achieve polar, semicrystalline thermoplastics that demonstrated adhesion properties over an order of magnitude greater than polyolefins. ${ }^{29}$ This method utilizes a BINOLbased phosphoric acid in combination with a titanium Lewis acid (1) to achieve isotactic PVEs with high degrees of stereoselectivity (up to 93\% meso diads). The identity of the alkyl side-chain functionality had a distinct impact on the thermal properties of the obtained polymers. Isotactic PVEs bearing linear alkyl substituents (e.g., ethyl, propyl, butyl) exhibited $T_{\mathrm{m}} \mathrm{s}$ that ranged from 65 to $76^{\circ} \mathrm{C}$, while those with branched side chains (e.g., isopropyl, isobutyl) featured $T_{\mathrm{m}} \mathrm{s}$ that ranged from 138 to $15^{\circ}{ }^{\circ} \mathrm{C}$. We envisioned leveraging this disparity of thermal properties between linear and branched vinyl ether substituents to prepare semicrystalline thermoplastics with tunable thermomechanical and interfacial properties. Herein, we present a systematic evaluation of stereoselective vinyl ether copolymerization using catalyst $\mathbf{1}$. We demonstrate the generality of this catalyst through the stereoselective statistical copolymerization of alkyl vinyl ether monomers. The introduction of comonomers does not influence the stereoselectivity of catalyst $\mathbf{1}$ and enables the realization of semicrystalline thermoplastics derived from polar vinyl monomers with tunable thermal properties. Furthermore, the functional group tolerance of 1 is exploited to access polar thermoplastics with rationally designed functionality.

Considering the disparate thermal properties exhibited by isotactic PVEs bearing linear and branched alkyl substituents, we chose to first explore the copolymerization of butyl vinyl ether (nBVE) with iBVE (Figure 2). Addition of a pre-chilled solution of $\mathbf{1}$ to a mixture of nBVE and iBVE at $-78{ }^{\circ} \mathrm{C}$ resulted in efficient copolymerization that produced high molecular weight materials $\left(M_{\mathrm{n}}>70 \mathrm{~kg}\right.$ $\left.\mathrm{mol}^{-1}\right)$. In order to tune the ultimate incorporation of nBVE in the resulting copolymers, reactions were performed using a variety of molar feed ratios of nBVE $\left(f_{\mathrm{Bu}}\right)$ relative to iBVE. As shown in Figure $3 \mathrm{~A}$, distinct ${ }^{1} \mathrm{H}$ NMR resonances were observed for $\operatorname{iBVE}(\delta 3.25-2.05 \mathrm{ppm}$, $\left.\mathrm{CDCl}_{3}\right)$ and $\mathrm{nBVE}\left(\delta 1.40-1.30 \mathrm{ppm}, \mathrm{CDCl}_{3}\right)$ repeat units, which were integrated relative to each other in order to determine the mole fraction of $\operatorname{nBVE}\left(F_{\mathrm{Bu}}\right)$. Analysis of the ${ }^{13} \mathrm{C}$ NMR enabled the determination of stereoselectivity by comparing the integration of the region corresponding to 
the racemo diads $\left(\delta 42.0-41.0 \mathrm{ppm}, \mathrm{CDCl}_{3}\right)$ to the region corresponding to the meso diads $(\delta 40.4-39.2 \mathrm{ppm}$, $\mathrm{CDCl}_{3}$ ) (Figure ${ }_{3} \mathrm{~B}$ ). Catalyst 1 resulted in poly(iBVE-conBVE) with high degrees of isotacticity (91-94\% meso diads) for all copolymer compositions studies, demonstrating the generality of $\mathbf{1}$ for simultaneous stereoselective polymerization of multiple alkyl vinyl ethers.

Next, we sought to investigate the relationship between $f_{\mathrm{Bu}}$ and $F_{\mathrm{Bu}}$ through kinetic analysis. Although values of $F_{\mathrm{Bu}}$ did not scale proportionally to $f_{\mathrm{Bu}}$, increasing $f_{\mathrm{Bu}}$ resulted in increased $F_{\mathrm{Bu}}$ which enabled the preparation of copolymers with pre-determined $F_{\mathrm{Bu}}$ values. In order to gain a deeper understanding of the reaction, a series of copolymerizations where $f_{\mathrm{Bu}}=0.50$ were quenched at various time points to evaluate reaction kinetics. As shown in Figure 4 , iBVE was consumed at a slower rate $\left(k_{\mathrm{obs}}=4.4 \times\right.$ $\left.10^{-4} \mathrm{~s}^{-1}\right)$ relative to the consumption of nBVE $\left(k_{\mathrm{obs}}=7.8 \times\right.$ $10^{-4} \mathrm{~s}^{-1}$ ) throughout the copolymerization. Previous explorations of vinyl ether copolymerization, in particular those initiated by trifluoromethanesulfonic acid or boron trifluoride diethyl etherate, observed the opposite reactivity trend, whereby the more sterically hindered comonomer was consistently incorporated at a faster rate..$^{30,31}$ The catalyst-controlled stereoselectivity exhibited in polymerizations mediated by 1 suggests a close interaction between the chiral anion and the propagating chain end, which we hypothesize to be interrupted by sterically demanding side chains. The relatively slow rate of iBVE consumption observed during copolymerization with nBVE is thus likely related to an adverse steric interaction between iBVE and 1. Comonomer consumption plateaus at a combined monomer conversion of $\sim 65 \%$ after $30 \mathrm{~min}$ (Figure $\mathrm{S}_{14}$ ) which, combined with the aforementioned rates, is consistent with the observed $F_{\mathrm{Bu}}$ values.

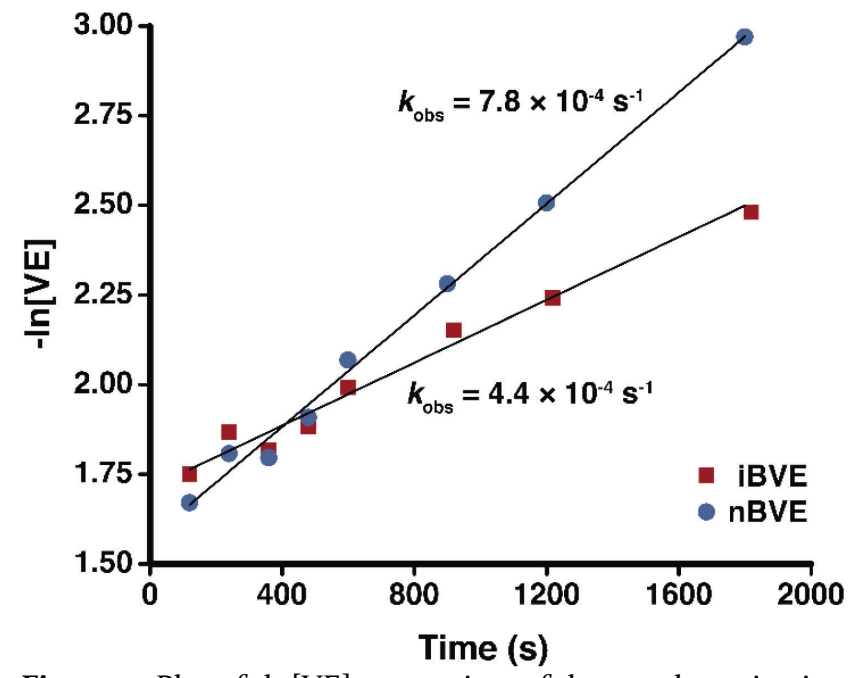

Figure 4. Plot of $-\ln [\mathrm{VE}]$ versus time of the copolymerization of iBVE and nBVE. Conversions of iBVE $(\boldsymbol{\bullet})$ and nBVE $(\bullet)$ monitored independently by ${ }^{1} \mathrm{H}$ NMR $\left(\mathrm{CDCl}_{3}\right)$. VE = vinyl ether. $[\mathrm{iBVE}]_{\mathrm{o}}=0.19 \mathrm{M} \cdot[\mathrm{nBVE}]_{\mathrm{o}}=0.19 \mathrm{M}$.

Each of the obtained poly(iBVE-co-nBVE) samples were semicrystalline thermoplastics at room temperature. As shown in Figure 5, differential scanning calorimetry (DSC) analysis at a scan rate of $10^{\circ} \mathrm{C} / \mathrm{min}$ with data taken from the second heating cycle revealed the copolymers
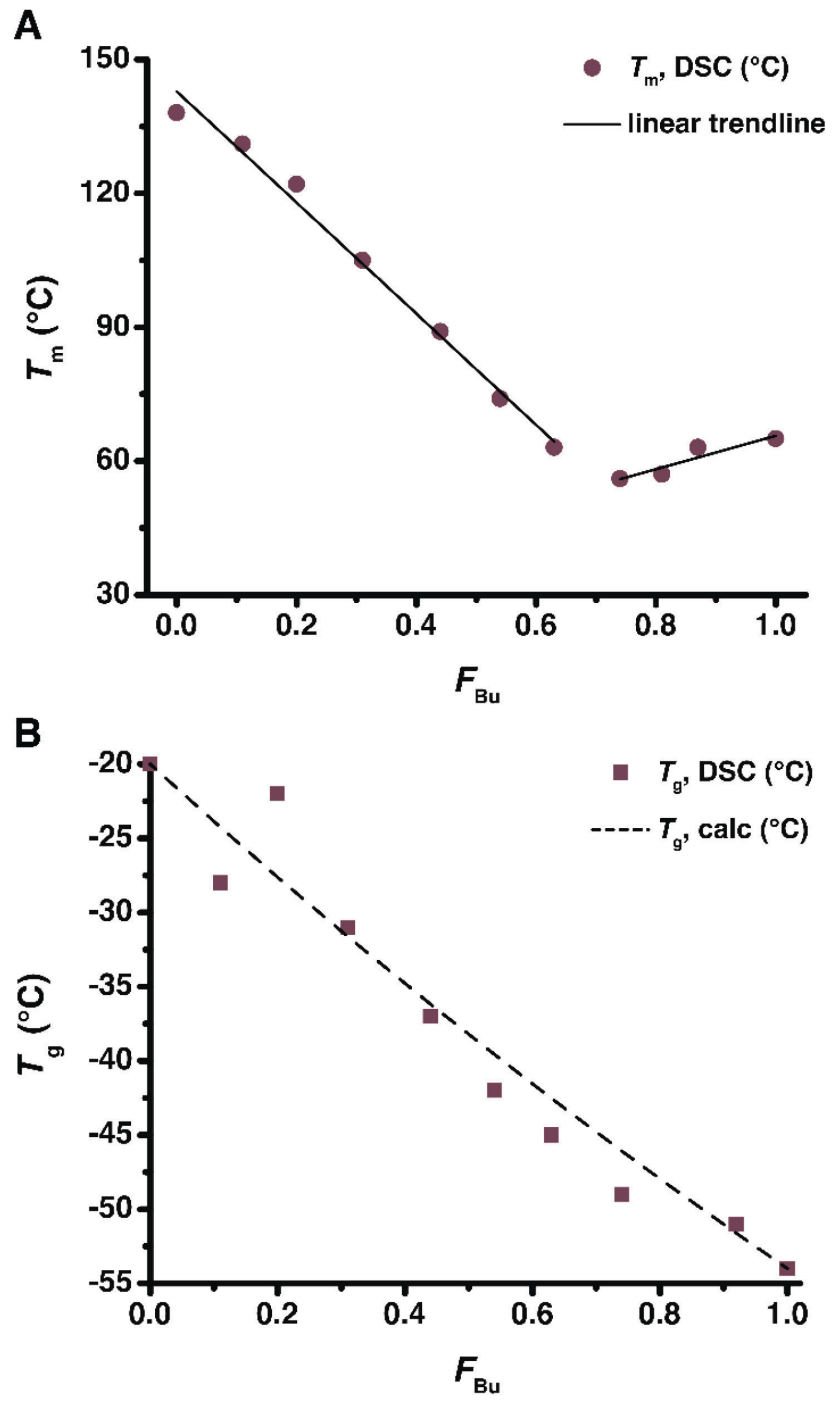

Figure 5. A) Plot of $T_{m}$ obtained by DSC as a function of molar incorporation of $\mathrm{nBVE}\left(\mathrm{F}_{\mathrm{Bu}}\right)$. Solid lines highlight observed trends. B) Plot of $\mathrm{T}_{\mathrm{g}}$ obtained by DSC as a function of $\mathrm{F}_{\mathrm{Bu}}$. Dashed line indicates $\mathrm{T}_{\mathrm{g}}$ values predicted using the Fox equation.

exhibited $T_{\mathrm{g}}$ and $T_{\mathrm{m}}$ values that span the range between those of poly(iBVE) $\left(T_{\mathrm{g}}=-20{ }^{\circ} \mathrm{C}, T_{\mathrm{m}}=138{ }^{\circ} \mathrm{C}\right)$ and poly (nBVE) $\left(T_{\mathrm{g}}=-53{ }^{\circ} \mathrm{C}, T_{\mathrm{m}}=65{ }^{\circ} \mathrm{C}\right)$. The $T_{\mathrm{g}}$ values observed by DSC scale with $F_{\mathrm{Bu}}$ as predicted by the Fox equation $^{32,33}$ (Figure $5^{\mathrm{B}}$ ), and remain well-below room temperature. The apparent $T_{\mathrm{m}}$ values decrease linearly with increasing $F_{\mathrm{Bu}}$ as expected, but reach an inflection point at approximately $F_{\mathrm{Bu}}=0.7$. At higher incorporations of nBVE (i.e., $F_{\mathrm{Bu}}>0.7$ ) a slight increase in $T_{\mathrm{m}}$ is observed, which we hypothesize is due to a switch in the composition of the major crystallites from iBVE repeat units to nBVE repeat units. Regardless, the observed trends afford the ability to rationally tune both $T_{\mathrm{g}}$ and $T_{\mathrm{m}}$ by selecting the appropriate $f_{\mathrm{Bu}}$ and highlight the general utility of vinyl ether copolymerizations facilitated by $\mathbf{1}$.

In an effort to explore the substrate scope of this methodology, we next performed a series of copolymerizations using ethyl vinyl ether (EVE) as a comonomer with iBVE. Similar to the iBVE/nBVE comonomer pair, utilizing a 


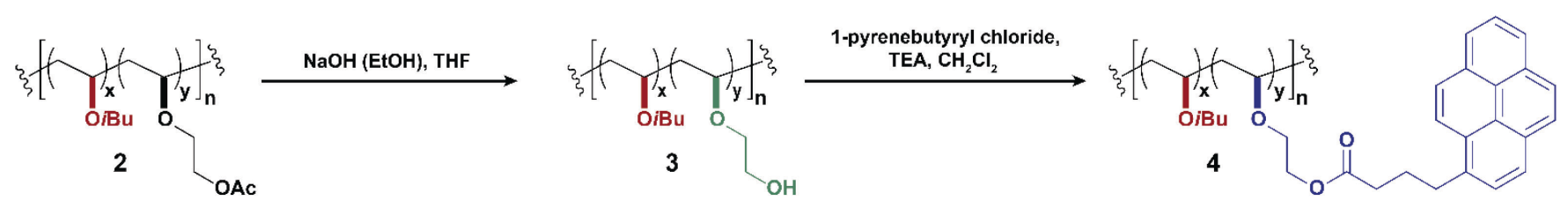

B

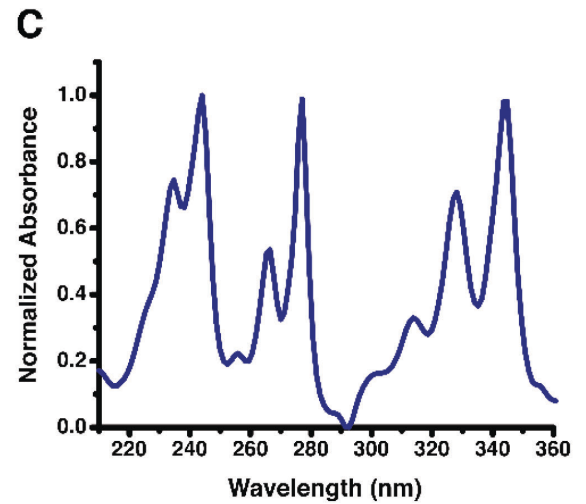

Figure 6. A) Reaction scheme depicting deprotection of copolymer 2 and post-functionalization of 3 to generate 4. B) Overlay of GPC traces before and after each step depicted in A. C) Photodiode array (PDA) trace at 13.9 min retention time (RT) confirming the structure of 4 .

variety of EVE molar feed ratios $\left(f_{\mathrm{Et}}\right)$ resulted in isotactic copolymers (91-93\% meso diads) with tunable degrees of EVE incorporation $\left(F_{\mathrm{Et}}\right)$ (Table $\left.\mathrm{S} \mathrm{s}\right)$. The obtained $F_{\mathrm{Et}}$ values were again consistently higher than $f_{\mathrm{Et}}$, likely due to a similar kinetic phenomenon to that described above. The $T_{\mathrm{g}}$ values exhibited by poly(iBVE-co-EVE) decreased as expected with increasing $F_{\mathrm{Et}}$, but appeared to plateau at $T_{\mathrm{g}}$ $=-37{ }^{\circ} \mathrm{C}$ when $F_{\mathrm{Et}} \geq 0.4$. Similarly, the observed $T_{\mathrm{m}}$ values decreased linearly with increasing $F_{\mathrm{Et}}$ and ranged from 138 ${ }^{\circ} \mathrm{C}$ to $50{ }^{\circ} \mathrm{C}$. C. No $T_{\mathrm{m}}$ was reliably observed in the second heating cycle by DSC when $F_{\mathrm{Et}} \geq 0.3$, although these materials crystallized slowly at room temperature and exhibited obvious first-order transitions in the first heating cycle.

Having demonstrated that the stereoselective copolymerization of nBVE or EVE with iBVE resulted in semicrystalline thermoplastic materials with modular $T_{\mathrm{g}}$ and $T_{\mathrm{m}}$ values, we next explored the diversity of functionality that can be incorporated into PVE copolymers. We prepared a copolymer using acyl-protected ethylene glycol vinyl ether (AcVE) as a comonomer with iBVE (2, Figure $6)$. Using a molar feed ratio $\left(f_{\text {Ac }}\right)$ of 0.05 we achieved a high molecular weight $\left(M_{\mathrm{n}}=41 \mathrm{~kg} \mathrm{~mol}^{-1}\right)$ copolymer with a $6.5 \%$ incorporation of $\mathrm{AcVE}\left(F_{\mathrm{Ac}}=0.065\right)$ and $90 \%$ meso diads. At this degree of incorporation, the material remained a high-melting thermoplastic with a $T_{\mathrm{g}}$ of $-26^{\circ} \mathrm{C}$ and a $T_{\mathrm{m}}$ of $124{ }^{\circ} \mathrm{C}$. Deprotection of the acyl functional group in 2 using $\mathrm{NaOH}$ efficiently yielded copolymer 3 which features repeat units containing free hydroxyl groups. As shown in Figure 6B, the gel permeation chromatography (GPC) traces for $\mathbf{2}$ and 3 closely overlap indicating the reaction proceeds without appreciable byproduct formation. Subsequent coupling with 1-pyrenebutyryl chloride yielded pyrene-appended copolymer 4, as evidenced by ${ }^{1} \mathrm{H}$ and ${ }^{13} \mathrm{C}$ NMR as well as GPC in conjunction with a photodiode array (PDA) detector (Figure 6C). This material exhibited a slightly-increased $T_{\mathrm{g}}$ of $-16{ }^{\circ} \mathrm{C}$ relative to 2 and remained a high-melting thermoplastic with a $T_{\mathrm{m}}$ of $126^{\circ} \mathrm{C}$, but was now fluorescent under UV irradiation.

In summary, we have leveraged the stereoselective cationic polymerization facilitated by catalyst $\mathbf{1}$ to prepare a series of isotactic vinyl ether-based copolymers. Through judicious choice of comonomer pairs, both the $T_{\mathrm{m}}$ and $T_{\mathrm{g}}$ of the resulting materials can be rationally tuned by modulating the relative incorporation of each comonomer. Furthermore, vinyl ethers which contain masked hydroxyl functionality were shown to be competent comonomers for further diversification and were utilized to append a fluorescent pyrene-derived functional group. The ability to copolymerize heteroatom-containing comonomers as well as perform multiple post-functionalization reactions without sacrificing the desirable thermal properties of the homopolymers represents a practical approach towards polar, high-performance thermoplastics and will lead to isotactic PVEs with a multitude of functionality.

\section{ASSOCIATED CONTENT}

The Electronic Supporting Information includes:

Experimental details, supplementary text, and additional figures S1-S28.

\section{AUTHOR INFORMATION}

\section{Corresponding Author}

*Frank A. Leibfarth; frankl@email.unc.edu

ORCID

Frank A. Leibfarth: oooo-ooo1-7737-0331

Aaron J. Teator: oooo-0oo3-4890-2487

Travis P. Varner: oooo-0oo3-1320-6262

Paige E. Jacky: oooo-0003-2375-4107

\section{Notes}

The authors declare no competing financial interest. 


\section{ACKNOWLEDGMENT}

This work is supported by the National Science Foundation under the CAREER award (CHE-1847362).

\section{REFERENCES}

(1) Aoshima, S.; Kanaoka, S. A Renaissance in Living Cationic Polymerization. Chem. Rev. 2009, 109, 5245-5287.

(2) Uchiyama, M.; Satoh, K.; Kamigaito, M. Cationic RAFT Polymerization Using Ppm Concentrations of Organic Acid. Angew. Chem. Int. Ed. 2015, 54, 1924-1928.

(3) Kottisch, V.; Michaudel, Q.; Fors, B. P. Photocontrolled Interconversion of Cationic and Radical Polymerizations. J. Am. Chem. Soc. 2017, 139, 10665-10668.

(4) Kottisch, V.; O’Leary, J.; Michaudel, Q.; Stache, E. E.; Lambert, T. H.; Fors, B. P. Controlled Cationic Polymerization: Single-Component Initiation under Ambient Conditions. J. Am. Chem. Soc. 2019, 141, 1060510609.

(5) Prasher, A.; Hu, H.; Tanaka, J.; Nicewicz, D. A.; You, W. Alcohol Mediated Degenerate Chain Transfer Controlled Cationic Polymerisation of Para -Alkoxystyrene. Polym. Chem. 2019, 10, 4126-4133.

(6) Kamigaito, M.; Satoh, K.; Uchiyama, M. Degenerative Chain-transfer Process: Controlling All Chain-growth Polymerizations and Enabling Novel Monomer Sequences. J. Polym. Sci. A Polym. Chem. 2019, 57, 243-254.

(7) Schröder, G. Poly(Vinyl Ethers). Ullmann's Encycl. Ind. Chem. 20oo, 28, 481-485.

(8) Fishbein, L.; Crowe, B. F. The Relation of Structure to Some Physical and Mechanical Properties of Poly(Vinyl Alkyl Ethers). Die Makromol. Chemie 1961, 48, 221-228.

(9) Dube, M. A.; Saldivar-Guerra, E.; Capata-Gonzalez, I. Copolymerization. Handb. Polym. Synth. Charact. Process. 2013, 105-125.

(10) Zhang, X.; Reyntjens, W.; Goethals, E. J. Living Copolymerization of Octadecyl Vinyl Ether with Isobutyl Vinyl Ether: Copolymerization Parameters and Crystallization Behaviour of the Copolymers. Polym. Int. 200o, 49, 277-280.

(11) Yoshida, T.; Seno, K.-I.; Kanaoka, S.; Aoshima, S. StimuliResponsive Reversible Physical Networks. I. Synthesis and Physical Network Properties of Amphiphilic Block and Random Copolymers with Long Alkyl Chains by Living Cationic Polymerization. J. Polym. Sci. A Polym. Chem. 2005, 43, 1155-1165.

(12) Yoshida, T.; Kanaoka, S.; Watanabe, H.; Aoshima, S. Stimuli-Responsive Reversible Physical Networks. II. Design and Properties of Homogeneous Physical Networks Consisting of Periodic Copolymers Synthesized by Living Cationic Polymerization. J. Polym. Sci. A Polym. Chem. 2005, 43, 2712-2722.

(13) Sugihara, S.; Kanaoka, S.; Aoshima, S. Double Thermosensitive Diblock Copolymers of Vinyl Ethers with Pendant Oxyethylene Groups: Unique Physical Gelation. Macromolecules 2005, 38, 1919-1927.

(14) Kieber, R. J.; Ozkardes, C.; Sanchez, N.; Kennemur, J. G. Cationic Copolymerization of Isosorbide towards ValueAdded Poly(Vinyl Ethers). Polym. Chem. 2019.

(15) Sugihara, S.; Kanaoka, S.; Aoshima, S. Thermosensitive Random Copolymers of Hydrophilic and Hydrophobic Monomers Obtained by Living Cationic Copolymerization. Macromolecules 2004, 37, 1711-1719.

(16) Yoshida, T.; Doi, M.; Kanaoka, S.; Aoshima, S. Polymer Surface Modification Using Diblock Copolymers Containing Azobenzene. J. Polym. Sci. A Polym. Chem. 2005, 43, 5704-5709.

(17) Ida, S.; Ouchi, M.; Sawamoto, M. Living Cationic Polymerization of an Azide-Containing Vinyl Ether toward Addressable Functionalization of Polymers. J. Polym. Sci. A
Polym. Chem. 2010, 48, 1449-1455.
(18) Yoshida, T.; Kanaoka, S.; Aoshima, S. Photo-Responsive Copolymers with Azobenzene Side Groups Synthesized by Living Cationic Polymerization: Efficient Amplification of Photosensitivity in Aqueous Photo-Switching System. J. Polym. Sci. A Polym. Chem. 2005, 43, 5337-5342.

Shibata, T.; Kanaoka, S.; Aoshima, S. Quantitative Synthesis of Star-Shaped Poly(Vinyl Ether)s with a Narrow Molecular Weight Distribution by Living Cationic Polymerization. J. Am. Chem. Soc. 2006, 128, 7497-7504.

Yoshida, T.; Kanaoka, S.; Aoshima, S. Photosensitive Copolymers with Various Types of Azobenzene Side Groups Synthesized by Living Cationic Polymerization. J. Polym. Sci. A Polym. Chem. 2005, 43, 4292-4297.

Goethals, E. J.; Reyntjens, W.; Lievens, S. Poly(Vinyl Ethers) as Building Blocks for New Materials. Macromol. Symp. 1998, 132, 57-64.

Kwon, S.; Lee, Y.; Mah, S. Living Cationic Polymerization of Vinyl Ethers in the Presence of Iodomethyl Methyl Ether and Zinc Iodide. Fibers Polym. 20o1, 2, 9-12.

Ittel, S. D.; Johnson, L. K.; Brookhart, M. Late-Metal Catalysts for Ethylene Homo- and Copolymerization. Chem. Rev. 2000, 100, 1169-1203.

Domski, G. J.; Rose, J. M.; Coates, G. W.; Bolig, A. D.; Brookhart, M. Living Alkene Polymerization: New Methods for the Precision Synthesis of Polyolefins. Prog. Polym. Sci. 2007, 32, 30-92.

Nakamura, A.; Anselment, T. M. J.; Claverie, J.; Goodall, B.; Jordan, R. F.; Mecking, S.; Rieger, B.; Sen, A.; Van Leeuwen, P. W. N. M.; Nozaki, K. Ortho-Phosphinobenzenesulfonate: A Superb Ligand for Palladium-Catalyzed CoordinationInsertion Copolymerization of Polar Vinyl Monomers. Acc. Chem. Res. 2013, 46, 1438-1449.

Franssen, N. M. G.; Reek, J. N. H.; de Bruin, B. Synthesis of Functional 'Polyolefins': State of the Art and Remaining Challenges. Chem. Soc. Rev. 2013, 42, 5809-5832.

Chen, E. Y.-X. Coordination Polymerization of Polar Vinyl Monomers by Single-Site Metal Catalysts. Chem. Rev. 2oo9, 109, 5157-5214.

Worch, J. C.; Prydderch, H.; Jimaja, S.; Bexis, P.; Becker, M. L.; Dove, A. P. Stereochemical Enhancement of Polymer Properties. Nat. Rev. Chem. 2019.

Teator, A. J.; Leibfarth, F. A. Catalyst-Controlled Stereoselective Cationic Polymerization of Vinyl Ethers. Science 2019, 363, 1439-1443.

Yuki, H.; Hatada, K.; Nagata, K.; Emura, T. Reactivities and ${ }_{13}$ C NMR Spectra of Alkyl Vinyl Ethers. Polym. J. 1970, 1, 269-270.

Higashimura, T.; Masamoto, J.; Okamura, S. Cationic Copolymerization of Vinyl Ethers Catalyzed by $\mathrm{BF}_{3} \cdot\left(\mathrm{OC}_{2} \mathrm{H}_{5}\right)$ 2. Kibunski Kagaku 1968, 25, 702-707.

Fox, T. G.; Flory, P. J. The Glass Temperature and Related Properties of Polystyrene. Influence of Molecular Weight. J. Polym. Sci. 1954, 14, 315-319. Temperatures and Related Properties of Polystyrene. I. Influence of Molecular Weight. J. Appl. Phys. 1950, 21, 581591. 


\section{Supplementary Materials for}

\section{Polar Thermoplastics with Tunable Physical Properties Enabled by the Stereoselective Copolymerization of Vinyl Ethers}

Aaron J. Teator, Travis P. Varner, Paige E. Jacky, Karolina A. Sheyko, and Frank A. Leibfarth*

Department of Chemistry, University of North Carolina Chapel Hill, Chapel Hill, NC 27599 (USA)

*Correspondence to: frank1@email.unc.edu

Table of Contents

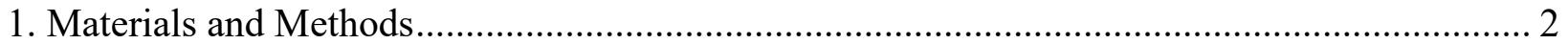

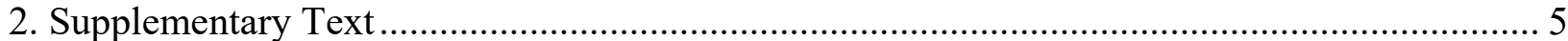

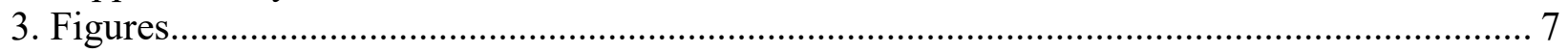

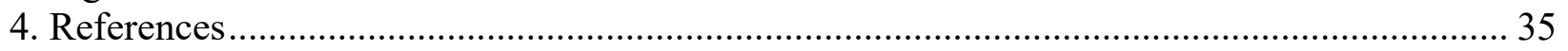




\section{Materials and Methods}

\subsection{General Considerations}

The compounds (R)-3,3'-bis(3,5-bis(trifluoromethyl)phenyl)-1,1'-binaphthyl phosphate, ${ }^{1}$ tetrachlorobis(tetrahydrofuran)titanium(IV) $\left(\mathrm{TiCl}_{4}(\mathrm{THF})_{2}\right),{ }^{2}$ and 1-pyrenebutyryl chloride ${ }^{3}$ were prepared according to literature procedures. Vinyl ether monomers were dried over $\mathrm{CaH}_{2}$ and distilled under vacuum prior to storage in a $\mathrm{N}_{2}$-filled glovebox for further use. All other reagents and solvents were purchased from commercial sources and used without further purification. Unless otherwise noted, solvents were dried and degassed using a Pure Process Technology solvent purification system and then subsequently stored over molecular sieves ( $3 \AA$ ). All syntheses were performed under inert atmosphere $\left(\mathrm{N}_{2}\right.$ or $\left.\mathrm{Ar}\right)$ using flame-dried or oven-dried glassware unless specified otherwise. NMR spectra were recorded using a Bruker DRX $400 \mathrm{MHz}$, Bruker AVANCE III $500 \mathrm{MHz}$, or Bruker AVANCE III $600 \mathrm{MHz}$ CryoProbe spectrometer. Chemical shifts $\delta(\mathrm{ppm})$ are referenced to tetramethylsilane (TMS) using the residual solvent as an internal standard $\left({ }^{1} \mathrm{H}\right.$ and $\left.{ }^{13} \mathrm{C}\right)$. For ${ }^{1} \mathrm{H}$ NMR: $\mathrm{CDCl}_{3}, 7.26$ ppm. For ${ }^{13} \mathrm{C} \mathrm{NMR:} \mathrm{CDCl}_{3}, 77.16 \mathrm{ppm}$. Coupling constants $(J)$ are expressed in hertz $(\mathrm{Hz})$.

\subsection{Macromolecular Characterization}

Gel permeation chromatography (GPC) was performed on a Waters 2695 separations module liquid chromatograph equipped with either four Waters Styragel HR columns (WAT044225, WAT044231, WAT044237, and WAT054460) arranged in series or two Agilent Resipore columns (PL1113-6300) maintained at $35{ }^{\circ} \mathrm{C}$, and a Waters 2414 refractive index detector at room temperature. Tetrahydrofuran was used as the mobile phase at a flow rate of $1.0 \mathrm{~mL} / \mathrm{min}$. Molecular weight and dispersity data are reported relative to polystyrene standards.

Melting-transition temperature $\left(T_{\mathrm{m}}\right)$ and glass-transition temperature $\left(T_{\mathrm{g}}\right)$ of precipitated and dried polymer samples were measured using differential scanning calorimetry (DSC) on a TA Instruments Discovery DSC. Unless specifically noted otherwise, values for $T_{\mathrm{m}}$ and $T_{\mathrm{g}}$ were obtained from a second heating scan after the thermal history was removed. All heating and cooling rates were $10{ }^{\circ} \mathrm{C} / \mathrm{min}$. Decomposition onset temperatures $\left(T_{\mathrm{d}}\right)$ of precipitated and dried polymer samples were measured by thermal gravimetric analysis (TGA) on a TA Instruments Q5000 Thermogravimetric Analyzer. Polymer samples were heated from ambient temperatures to $600{ }^{\circ} \mathrm{C}$ at a heating rate of $10{ }^{\circ} \mathrm{C} / \mathrm{min}$. Values of $T_{\mathrm{d}}$ (temperature at $5 \%$ weight loss) were obtained from wt $\%$ vs. temperature $\left({ }^{\circ} \mathrm{C}\right)$ plots.

\subsection{Syntheses and Characterization Data}

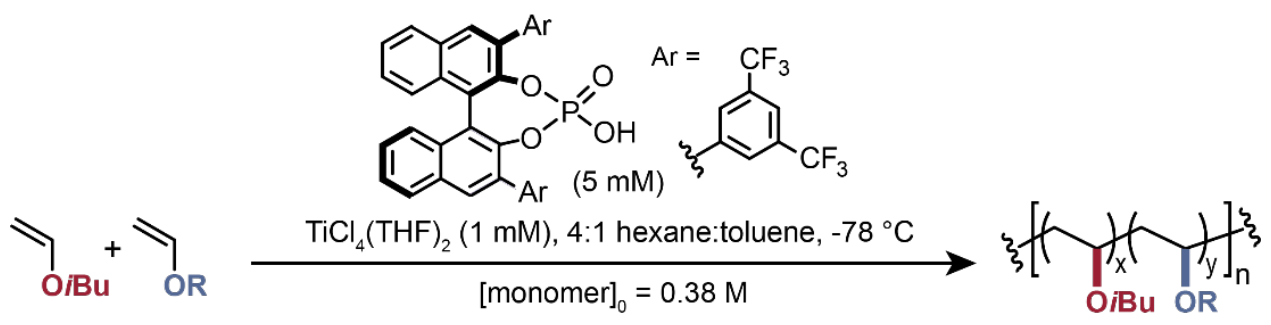

\section{General copolymerization procedure}

Copolymerizations were performed in septum-capped reaction vials prepared in a $\mathrm{N}_{2}$-filled glovebox. An oven-dried $8 \mathrm{~mL}$ septum-capped vial equipped with a stir bar was charged with isobutyl vinyl ether (iBVE), comonomer, and hexane. In this vial: the total monomer concentration was kept constant at $0.38 \mathrm{M}$ and the total solution volume was kept constant at $1.62 \mathrm{~mL}$. A separate 
$8 \mathrm{~mL}$ septum-capped vial equipped with a stir bar was charged with $0.26 \mathrm{~mL}$ of a $0.05 \mathrm{M}$ stock solution of (R)-3,3'-bis(3,5-bis(trifluoromethyl)phenyl)-1,1'-binaphthyl phosphate in $\mathrm{MePh}$ $(0.013 \mathrm{mmol})$ and $0.26 \mathrm{~mL}$ of a $0.01 \mathrm{M}$ stock solution of $\mathrm{TiCl}_{4}(\mathrm{THF})_{2}$ in $\mathrm{MePh}(0.0026 \mathrm{mmol})$. Both vials were removed from the glove box and cooled to $-78^{\circ} \mathrm{C}$ in a dry ice/acetone bath. After stirring at $-78{ }^{\circ} \mathrm{C}$ for $15 \mathrm{~min}$, the entire $\mathrm{MePh}$ solution was transferred via syringe to the vial containing monomer solution. The reaction was stirred at $-78^{\circ} \mathrm{C}$ for $2 \mathrm{~h}$, after which time $1.0 \mathrm{~mL}$ of $\mathrm{Et}_{3} \mathrm{~N} / \mathrm{MeOH}$ solution $(5 \% v / v)$ was added to quench the polymerization. Upon warming to room temperature, the mixture was washed with $1 \mathrm{~N} \mathrm{HCl}$ and all volatiles removed in vacuo. The crude polymer was dissolved in 1-2 $\mathrm{mL} \mathrm{CH}_{2} \mathrm{Cl}_{2}$ and filtered through a plug of $\mathrm{SiO}_{2}(4-5 \mathrm{~cm})$ in a glass pipette eluting with additional $\mathrm{CH}_{2} \mathrm{Cl}_{2}$. The resulting purified polymer was dried under vacuum for at least $12 \mathrm{~h}$ to a constant weight.

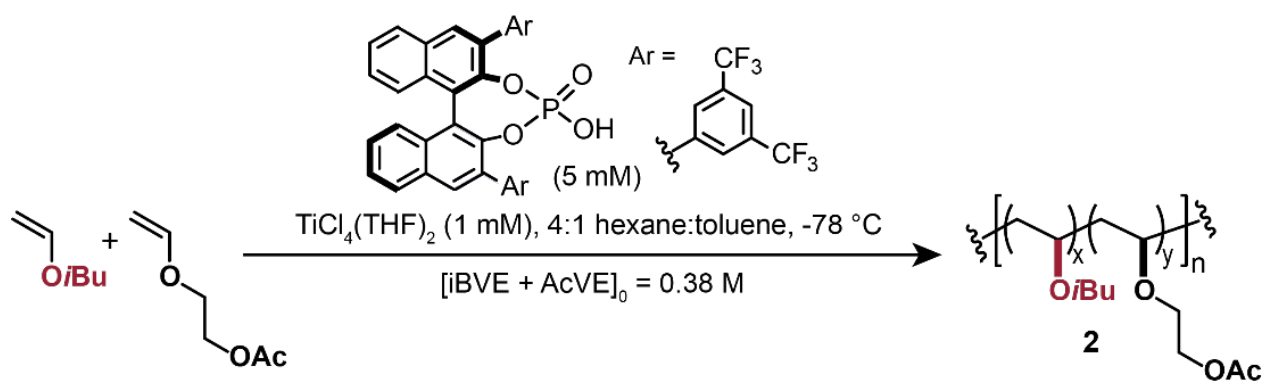

\section{Copolymerization of iBVE with acyl-protected ethylene glycol vinyl ether (AcVE)}

An oven-dried $20 \mathrm{~mL}$ septum-capped vial equipped with a stir bar was charged with iBVE $(0.29 \mathrm{~mL}, 2.10 \mathrm{mmol}), \operatorname{AcVE}(15 \mathrm{mg}, 0.11 \mathrm{mmol})$, and hexane $(4.22 \mathrm{~mL})$. A separate $8 \mathrm{~mL}$ septum-capped vial equipped with a stir bar was charged with $0.6 \mathrm{~mL}$ of a $0.05 \mathrm{M}$ stock solution of $(R)$-3,3'-bis(3,5-bis(trifluoromethyl)phenyl)-1,1'-binaphthyl phosphate in $\mathrm{MePh}(0.03 \mathrm{mmol})$ and $0.6 \mathrm{~mL}$ of a $0.01 \mathrm{M}$ stock solution of $\mathrm{TiCl}_{4}(\mathrm{THF})_{2}$ in $\mathrm{MePh}(0.006 \mathrm{mmol})$. Both vials were removed from the glove box and cooled to $-78^{\circ} \mathrm{C}$ in a dry ice/acetone bath. After stirring at -78 ${ }^{\circ} \mathrm{C}$ for $15 \mathrm{~min}$, the entire $\mathrm{MePh}$ solution was transferred via syringe to the vial containing monomer solution. The reaction was stirred at $-78^{\circ} \mathrm{C}$ for $4 \mathrm{~h}$, after which time $3.0 \mathrm{~mL}$ of $\mathrm{Et}_{3} \mathrm{~N} / \mathrm{MeOH}$ solution $(5 \% \mathrm{v} / \mathrm{v})$ was added to quench the polymerization. Upon warming to room temperature, the mixture was washed with $1 \mathrm{~N} \mathrm{HCl} \mathrm{and} \mathrm{all} \mathrm{volatiles} \mathrm{removed} \mathrm{in} \mathrm{vacuo.} \mathrm{The} \mathrm{crude}$ polymer was dissolved in 1-2 $\mathrm{mL} \mathrm{CH}_{2} \mathrm{Cl}_{2}$ and filtered through a short $(5-6 \mathrm{~cm})$ column (diameter $=16 \mathrm{~mm}$ ) of $\mathrm{SiO}_{2}$ eluting with additional $\mathrm{CH}_{2} \mathrm{Cl}_{2}$. The resulting purified polymer was dried under vacuum for at least $12 \mathrm{~h}$ to a constant weight. Yield: $46 \%$.

$f_{\mathrm{Ac}}=0.05 ; F_{\mathrm{Ac}}=0.065 .{ }^{1} \mathrm{H} \mathrm{NMR}\left(400 \mathrm{MHz}, \mathrm{CDCl}_{3}\right): \delta 4.16$ (t), 3.62 (br.), 3.56 (br.), 3.47 (br.), 3.25 (br.), 3.16 (br.), 2.07 (s), 1.82 (br.), 1.78 (septet), 1.53 (br.), 0.88 (d). ${ }^{13} \mathrm{C}$ NMR (125 MHz): $\delta 171.1,75.9,73.9,41.6,39.7,39.4,29.1,19.8$. GPC: $M_{\mathrm{n}}=41 \mathrm{kDa} ; \nexists=1.9$. DSC: $T_{\mathrm{g}}=-26^{\circ} \mathrm{C}$, $T_{\mathrm{m}}=124^{\circ} \mathrm{C}$.
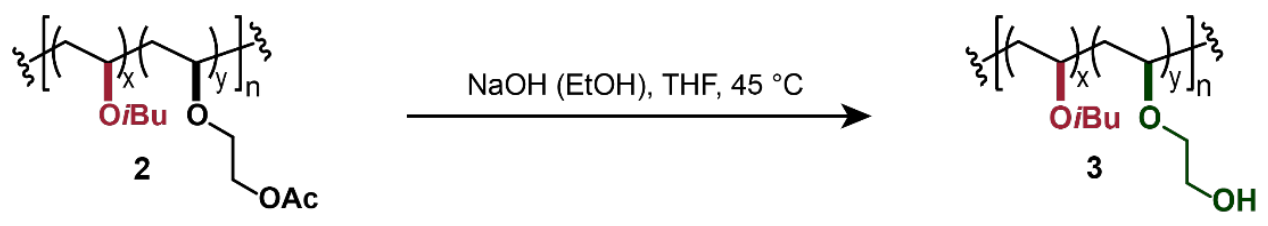

Hydrolysis of copolymer 2 
An oven-dried $8 \mathrm{~mL}$ septum-capped vial equipped with a stir bar was charged with copolymer 2 ( $0.215 \mathrm{~g}, 0.136 \mathrm{mmol}$ AcVE repeat units) and dry THF (2.5 mL). NaOH (55 mg, $1.38 \mathrm{mmol})$, dissolved in EtOH $(0.30 \mathrm{~mL})$, injected into vial containing copolymer solution and mixture heated to $45{ }^{\circ} \mathrm{C}$. After 16 hours, mixture cooled to RT and directly poured into ice-cold $\mathrm{H}_{2} \mathrm{O}$. Pale yellow precipitate collected by filtration and subsequently triturated with excess $\mathrm{MeOH}$ by vigorously stirring for $30 \mathrm{~min}$. The resulting material was dissolved in THF (1-2 mL) and precipitated into ice cold $\mathrm{H}_{2} \mathrm{O}$ a second time. The pure, white copolymer was collected by filtration and dried under vacuum for at least $12 \mathrm{~h}$ to a constant weight. Yield: $140 \mathrm{mg}$ (77\%).

${ }^{1} \mathrm{H}$ NMR (400 MHz, $\mathrm{CDCl}_{3}$ ): $\delta 3.66$ (br.), 3.47 (br.), 3.15 (br.), 1.82 (br.), 1.77 (septet), 1.53 (br.), 0.88 (d). GPC: $M_{\mathrm{n}}=37 \mathrm{kDa} ; Ð=2.1$. DSC: $T_{\mathrm{g}}=-16^{\circ} \mathrm{C}, T_{\mathrm{m}}=131^{\circ} \mathrm{C}$.
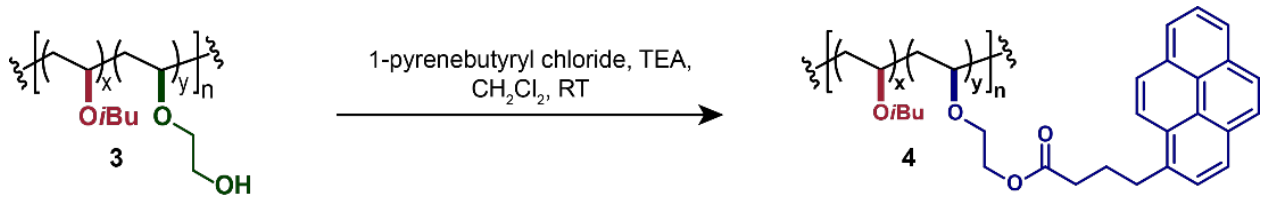

Functionalization of copolymer 3 with 1-pyrenebutyryl chloride

An oven-dried $8 \mathrm{~mL}$ septum-capped vial equipped with a stir bar was charged with copolymer 3 (0.040 g, $0.026 \mathrm{mmol}$ hydroxyl repeat units) and dry $\mathrm{CH}_{2} \mathrm{Cl}_{2}(5.0 \mathrm{~mL})$. Triethylamine $(18 \mu \mathrm{L}, 0.130 \mathrm{mmol})$ added, followed by a solution of 1-pyrenebutyryl chloride $(0.040 \mathrm{~g}, 0.130 \mathrm{mmol})$ in dry $\mathrm{CH}_{2} \mathrm{Cl}_{2}(1.0 \mathrm{~mL})$. Mixture stirred at room temperature (RT) for 3 hours, at which point it was directly poured into ice-cold $\mathrm{MeOH}$. Pale yellow precipitate collected by filtration, dissolved in THF $(1 \mathrm{~mL})$, precipitated into ice cold $\mathrm{MeOH}$ a second time. The pure, pale yellow copolymer was collected by filtration and dried under vacuum for at least $12 \mathrm{~h}$ to a constant weight. Yield: $33 \mathrm{mg}$ (70\%). Note: GPC analysis with photodiode array (PDA) detection $(344 \mathrm{~nm})$ confirms the presence of pyrene in copolymer 4.

${ }^{1} \mathrm{H}$ NMR (400 MHz, $\mathrm{CDCl}_{3}$ ): $\delta 8.30$ (br.), 8.16 (br.), 8.10 (br.), 8.02 (br.), 7.87 (br.), 4.21 (br.), 3.57 (br.), 3.48 (br.), 3.20 (br.), 3.15 (br.), 2.51 (br.), 2.18 (br.), 1.83 (br.), 1.77 (septet), 1.53 (br.), 0.88 (d). ${ }^{13} \mathrm{C}$ NMR (125 MHz): $\delta$ 173.37, 131.4, 130.9, 130.0, 128.7, 127.5, 127.3, 126.7, 125.8, 125.1, 124.9, 123.4, 75.8, 73.8, 41.4, 39.6, 39.3, 28.9, 19.7. GPC: $M_{\mathrm{n}}=47 \mathrm{kDa} ; Ð=2.3$. DSC: $T_{\mathrm{g}}=-16^{\circ} \mathrm{C}, T_{\mathrm{m}}=126^{\circ} \mathrm{C}$. 


\section{Supplementary Text}

\subsection{Copolymerization of ethyl vinyl ether (EVE) with iBVE}

Table S1. Summary of copolymerization experiments with iBVE and EVE using 1.

\begin{tabular}{|c|c|c|c|c|c|c|}
\hline$f_{\mathrm{Et}}{ }^{a}$ & $F_{\mathrm{Et}^{\mathrm{b}}}^{\mathrm{b}}$ & $M_{\mathrm{n}}\left(\mathrm{kg} \mathrm{mol}^{-1}\right)^{\mathrm{C}}$ & $\bigoplus^{c}$ & $m(\%)$ & $T_{\mathrm{g}}\left({ }^{\circ} \mathbf{C}\right)^{\mathrm{d}}$ & $T_{m}\left({ }^{\circ} \mathrm{C}\right)^{d}$ \\
\hline 0.05 & 0.09 & 58 & 1.7 & 93 & -26 & 132 \\
\hline 0.10 & 0.13 & 58 & 1.9 & 93 & -25 & 132 \\
\hline 0.15 & 0.22 & 62 & 1.9 & 93 & -29 & 107 \\
\hline 0.20 & 0.34 & 83 & 2.0 & 92 & -32 & 66 \\
\hline 0.30 & 0.38 & 101 & 2.4 & 93 & -33 & $50^{e}$ \\
\hline 0.40 & 0.52 & 41 & 1.7 & 93 & -37 & $39^{e}$ \\
\hline 0.50 & 0.62 & 90 & 2.7 & 92 & -37 & $40^{\mathrm{e}}$ \\
\hline 0.65 & 0.74 & 66 & 2.0 & 91 & -37 & not observed \\
\hline 0.8 & 0.83 & 51 & 1.8 & 93 & -38 & $41^{\mathrm{e}}$ \\
\hline 0.9 & 0.91 & 82 & 2.6 & 92 & -39 & $42^{\mathrm{e}}$ \\
\hline
\end{tabular}

${ }^{\mathrm{a}}$ mole fraction of EVE in the monomer feed. ${ }^{\mathrm{b}}$ mole fraction of EVE in copolymer determined by $1 \mathrm{H}$ NMR integration.

${ }^{\mathrm{c}} M_{\mathrm{n}}$ indicates the number average molecular weight of the polymer. Dispersity was calculated according to $Đ=M_{\mathrm{w}} / M_{\mathrm{n}}$ where $M_{\mathrm{w}}$ is the weight average molecular weight. ${ }^{\mathrm{d}} T_{\mathrm{g}}$ and $T_{\mathrm{m}}$ obtained from a second heating scan $\left(10^{\circ} \mathrm{C} / \mathrm{min}\right)$ after the thermal history was removed. ${ }^{\mathrm{e}} T_{\mathrm{m}}$ values obtained from first heating scan $\left(10{ }^{\circ} \mathrm{C} / \mathrm{min}\right)$ after annealing at room temperature for several days. 


\subsection{Pseudo-First Order Kinetic Analyses}

The cationic polymerization of vinyl ethers, including iBVE, nBVE, EVE, and AcVE, facilitated by catalyst $\mathbf{1}$ may be represented as:

$$
M+C \longrightarrow P
$$

Under the assumption that there are negligible side-reactions taking place, any of the above listed monomers (M) will always be present in a large excess over the catalyst (C). As such, pseudofirst-order kinetics are valid for rate calculations. In this scenario, the following rate law applies:

$$
\frac{d[P]}{d t}=k[M][C]_{0}=k[M]
$$

The integrated form of the rate equation is represented as:

$$
\ln [M]=\ln [M]_{0}-k t
$$

This equation indicates that plotting inverse $\ln [\mathrm{M}]$ versus $t(\mathrm{~s})$ should give a linear plot where $k$ is equal to the slope of the line. Figure 4 in the main text illustrates this relationship.

During the copolymerization of $\mathrm{BBVE}$ and nBVE, significant overlap occurs in the ${ }^{1} \mathrm{H}$ NMR spectrum that hinders the ability to monitor the relative consumption of each monomer independently. Peak deconvolution of the vinyl region $\left(\delta 6.40-6.48 \mathrm{ppm}, \mathrm{CDCl}_{3}\right)$ using OriginPro $8^{4}$ in the presence of an internal standard (i.e., 1,4-dimethoxybenzene), however, enables the accurate determination of individual monomer concentration at various time points (Table S1). A representative example can be seen in Figure S13.

Table S2. Summary of peak deconvolution data obtained from a copolymerization of nBVE with iBVE with $f_{\mathrm{Bu}}=0.50$ that was quenched at $20 \mathrm{~min}(t=1200 \mathrm{~s})$. Peak denominations correspond to those seen in Figure S13.

\begin{tabular}{cccc}
\hline Monomer & Peak & Area & Total Area \\
\hline \multirow{3}{*}{ iBVE } & 1 & $9.96 \mathrm{E}-5$ & \\
& 2 & $1.10 \mathrm{E}-4$ & $4.26 \mathrm{E}-4$ \\
& 3 & $1.12 \mathrm{E}-4$ & \\
& 4 & $1.10 \mathrm{E}-4$ & \\
& 5 & $7.58 \mathrm{E}-5$ & \\
nBVE & 6 & $7.98 \mathrm{E}-5$ & \multirow{2}{*}{$3.00 \mathrm{E}-4$} \\
& 7 & $8.10 \mathrm{E}-5$ & \\
\hline & 8 & $7.14 \mathrm{E}-5$ & \\
\hline
\end{tabular}




\section{Figures}

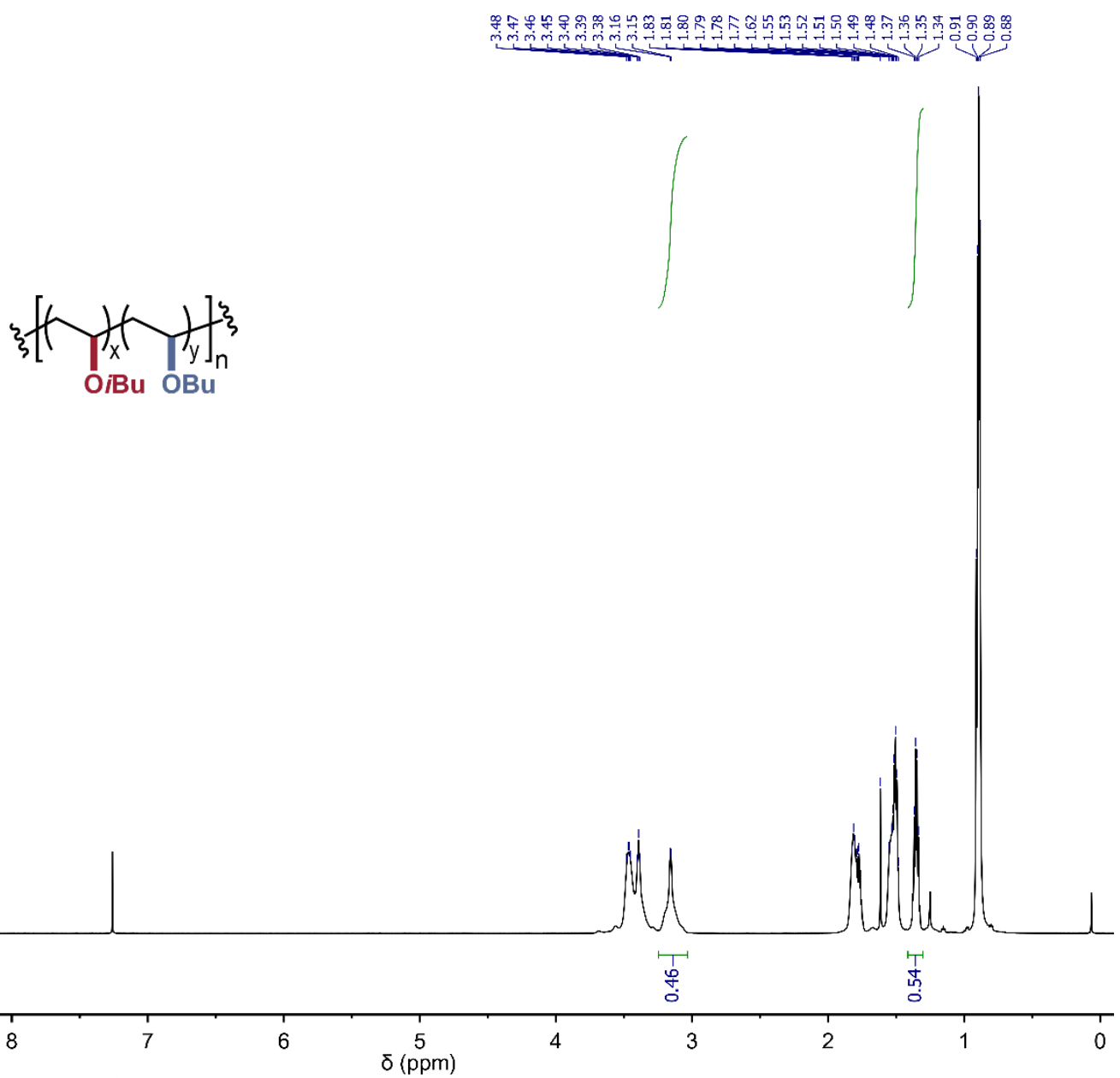

Figure S1. Representative ${ }^{1} \mathrm{H}$ NMR spectrum of isotactic poly(iBVE-co-nBVE). In this example, $F_{\mathrm{Bu}}=0.54\left(\mathrm{CDCl}_{3}\right)$. 


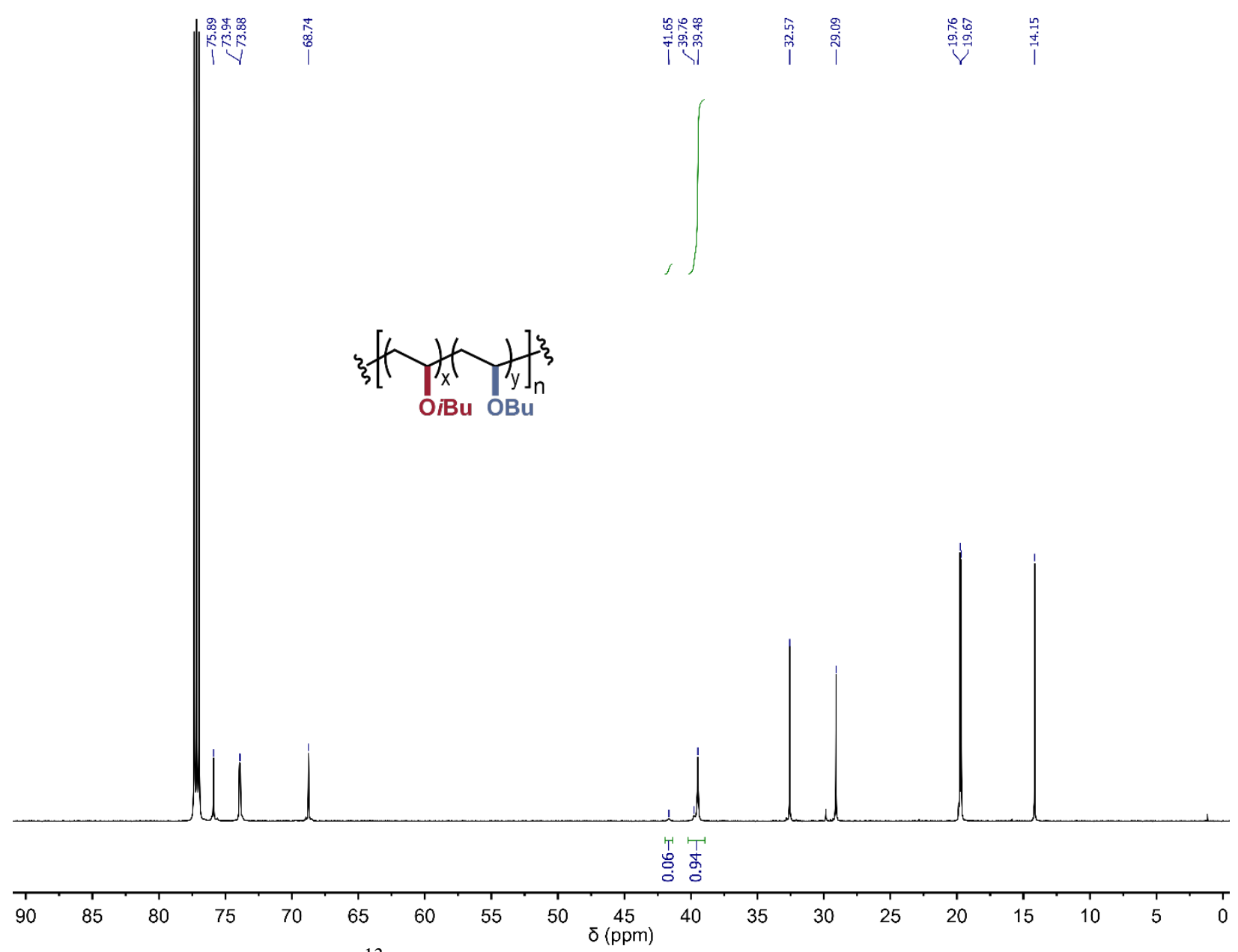

Figure S2. Representative ${ }^{13} \mathrm{C}$ NMR spectrum of isotactic poly(iBVE-co-nBVE) with relative integrations amounting to $94 \%$ meso diads. In this example, $F_{\mathrm{Bu}}=0.54\left(\mathrm{CDCl}_{3}\right)$. 


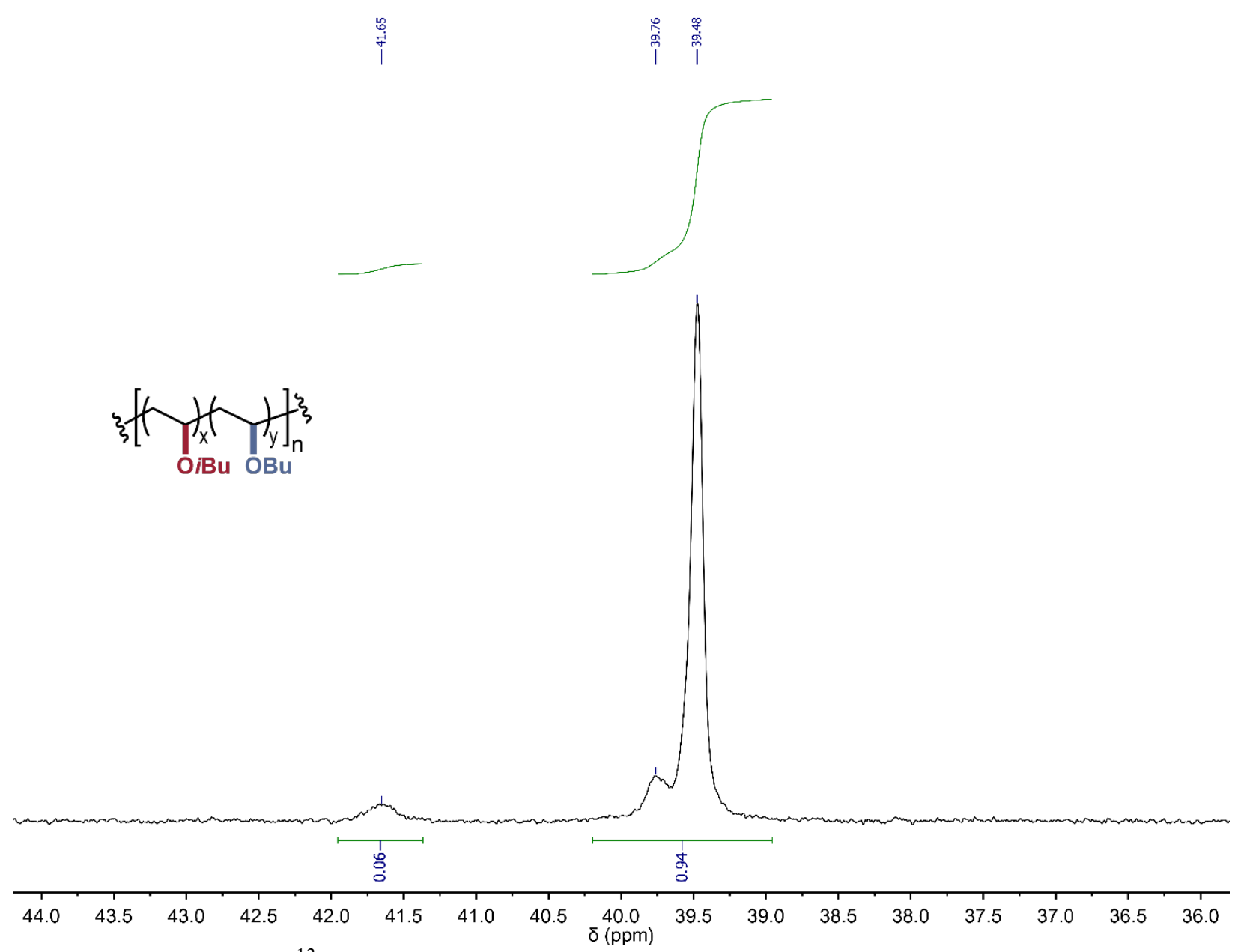

Figure S3. Expanded ${ }^{13} \mathrm{C}$ NMR spectrum of a representative sample of isotactic poly(iBVE-co$\mathrm{nBVE}$ ) showing relative integrations amounting to $94 \%$ meso diads. In this example, $F_{\mathrm{Bu}}=0.54$ $\left(\mathrm{CDCl}_{3}\right)$. 


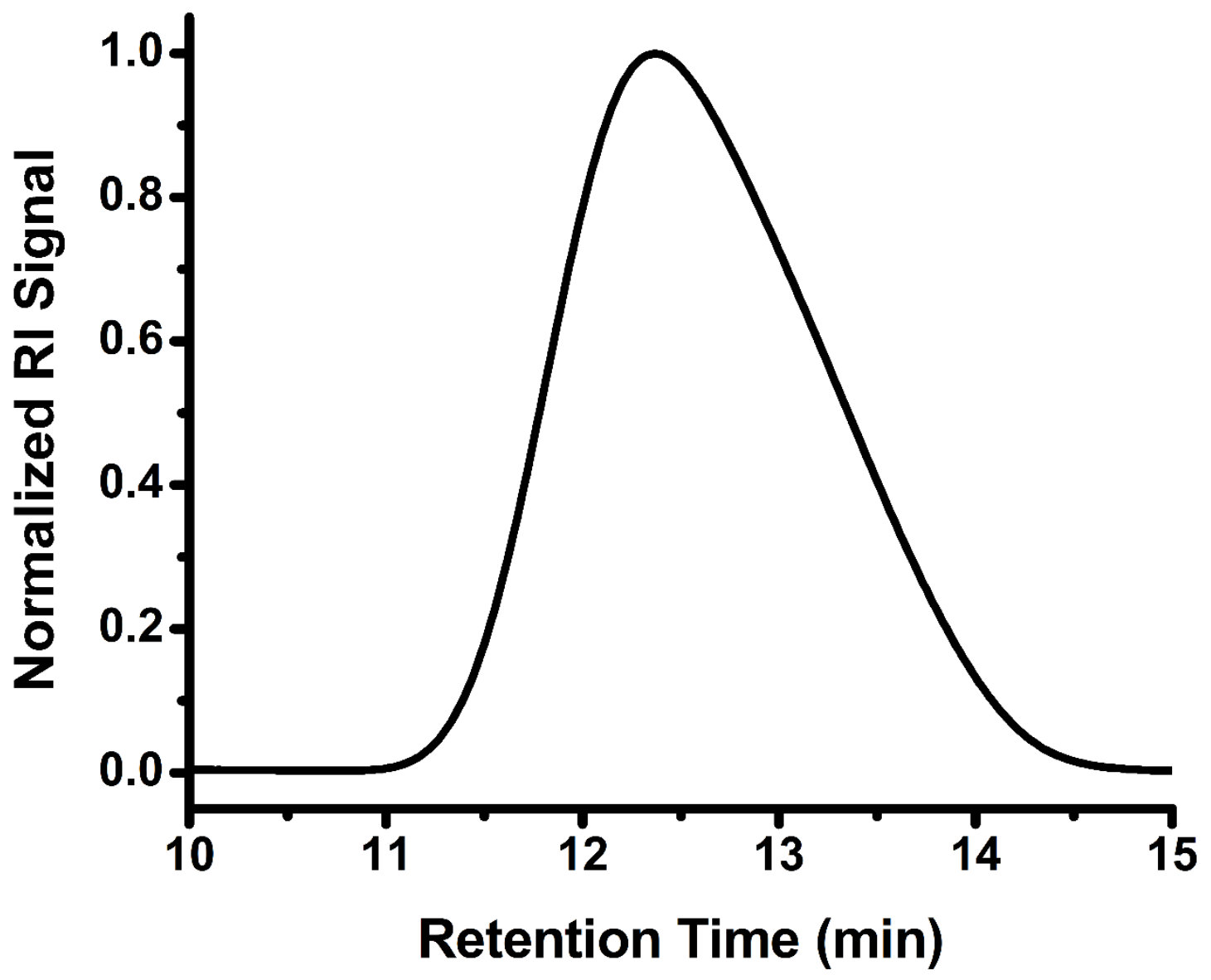

Figure S4. Representative GPC RI trace of isotactic poly(iBVE-co-nBVE). In this example, $F_{\mathrm{Bu}}$ $=0.54$. 


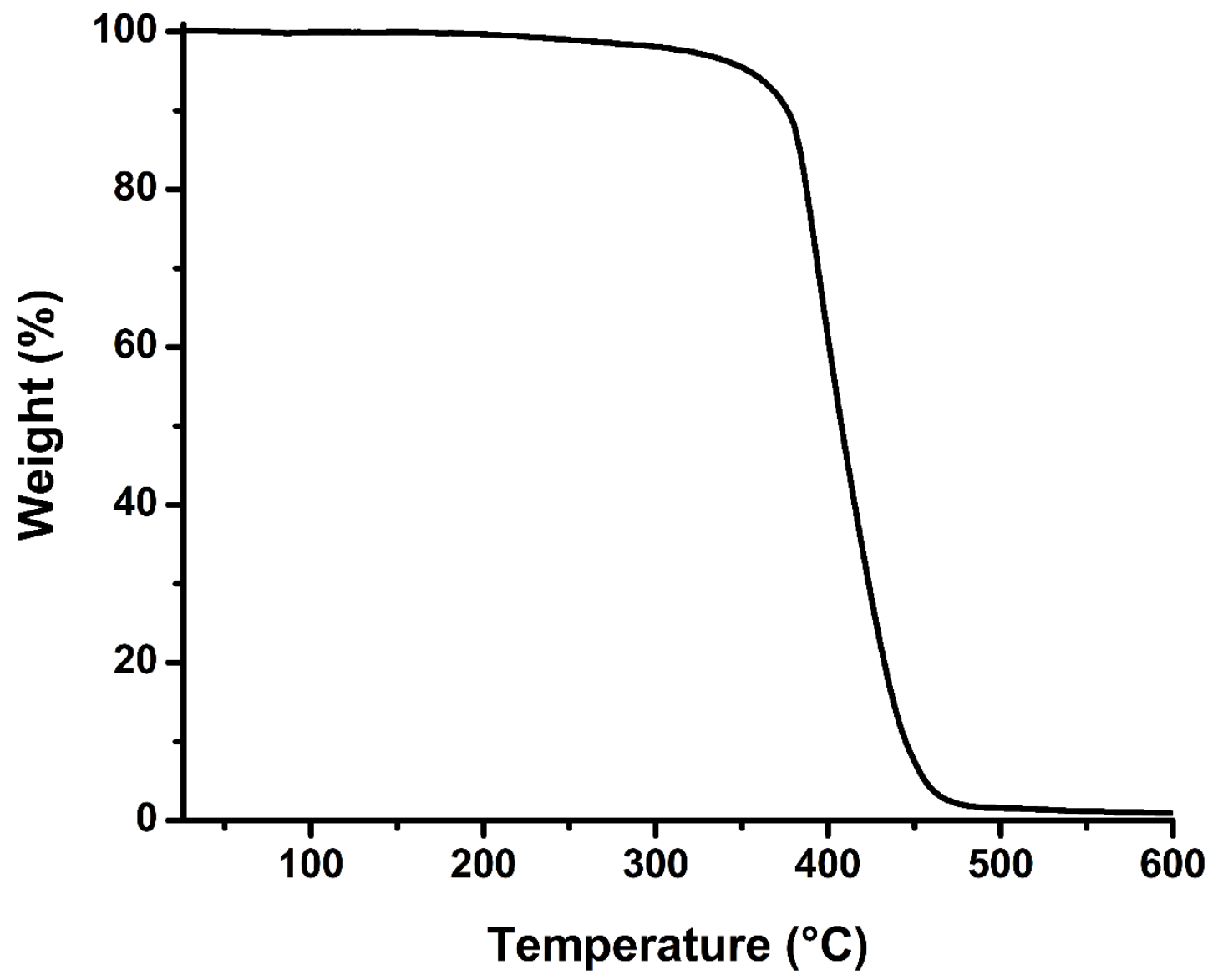

Figure S5. Representative TGA curve for isotactic poly(iBVE-co-nBVE). In this example, $F_{\mathrm{Bu}}=$ 0.54 . 


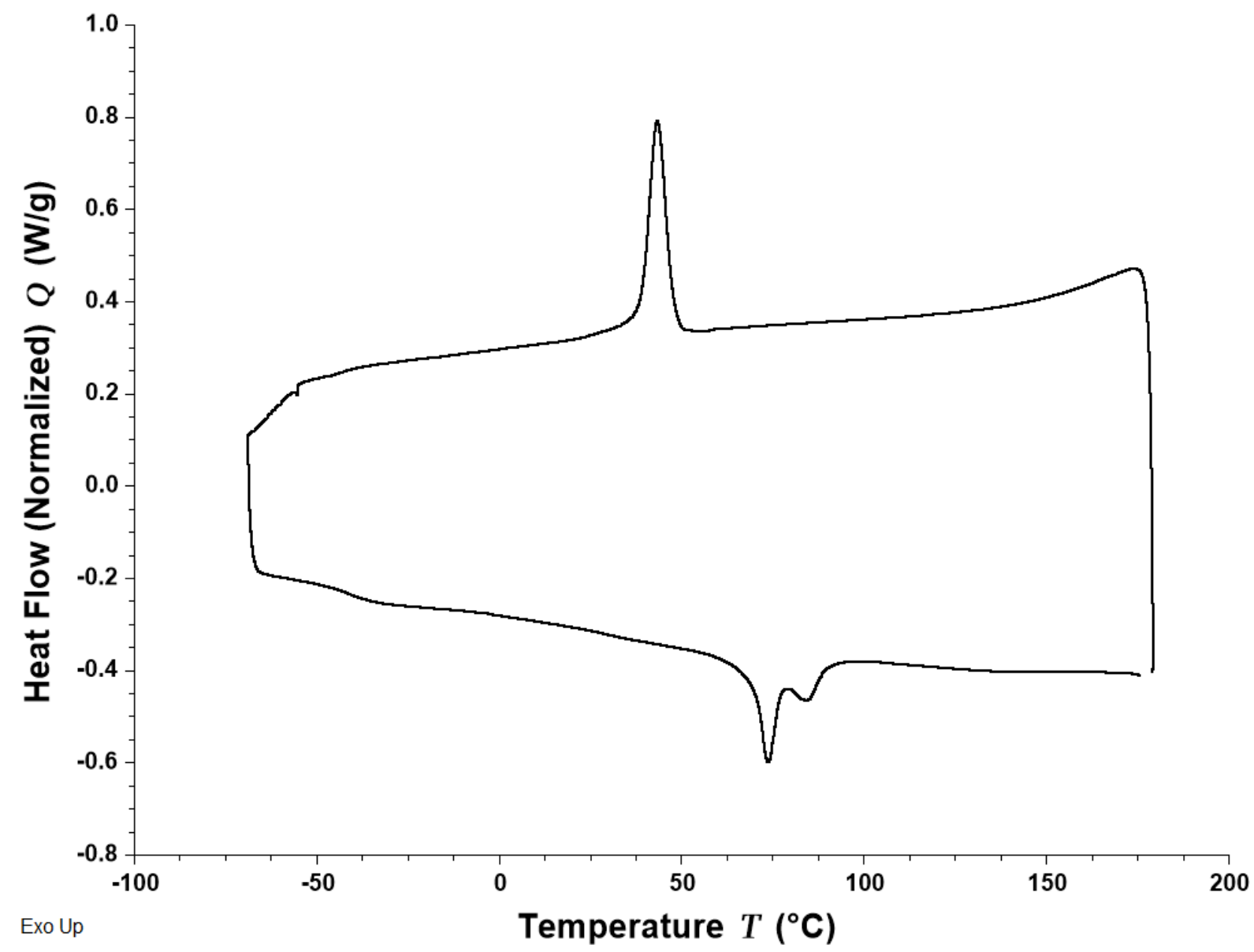

Figure S6. Representative DSC curves for isotactic poly(iBVE-co-nBVE). In this example, $F_{\mathrm{Bu}}=$ 0.54 . 


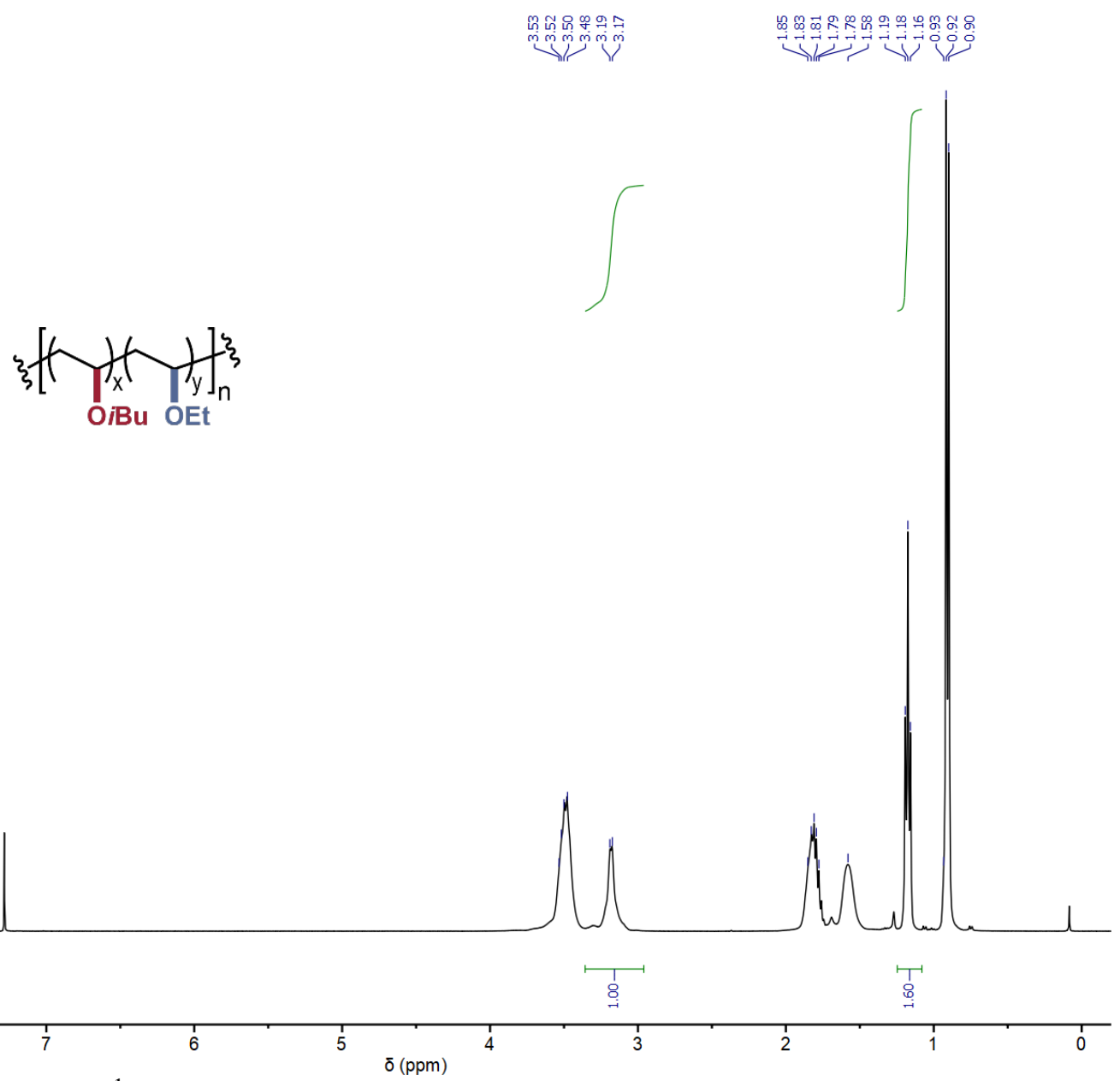

Figure S7. Representative ${ }^{1} \mathrm{H}$ NMR spectrum of isotactic poly(iBVE-co-EVE). In this example, $F_{\mathrm{Et}}=0.52\left(\mathrm{CDCl}_{3}\right)$. 


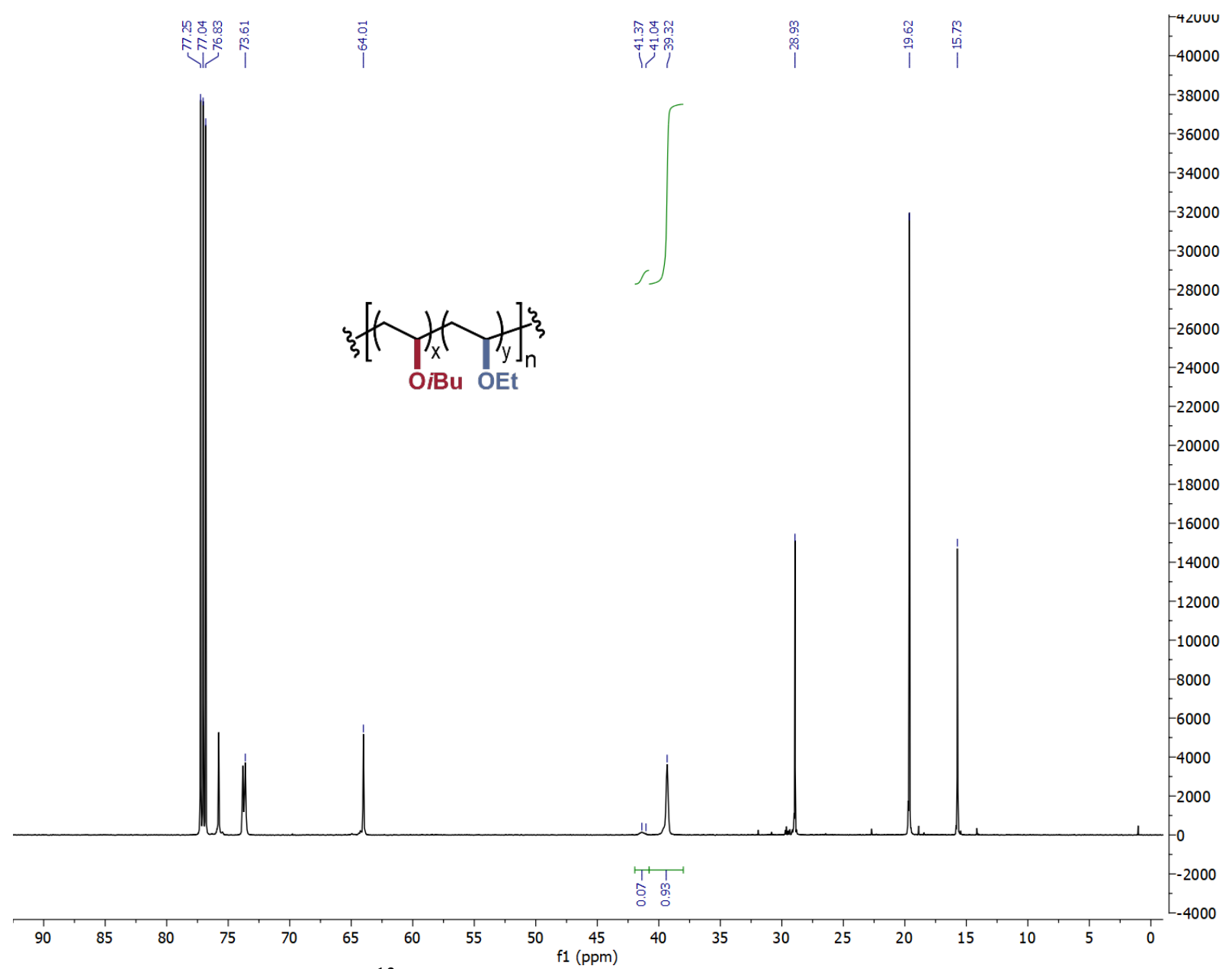

Figure S8. Representative ${ }^{13} \mathrm{C}$ NMR spectrum of isotactic poly(iBVE-co-EVE) with relative integrations amounting to $93 \%$ meso diads. In this example, $F_{\mathrm{Et}}=0.52\left(\mathrm{CDCl}_{3}\right)$. 


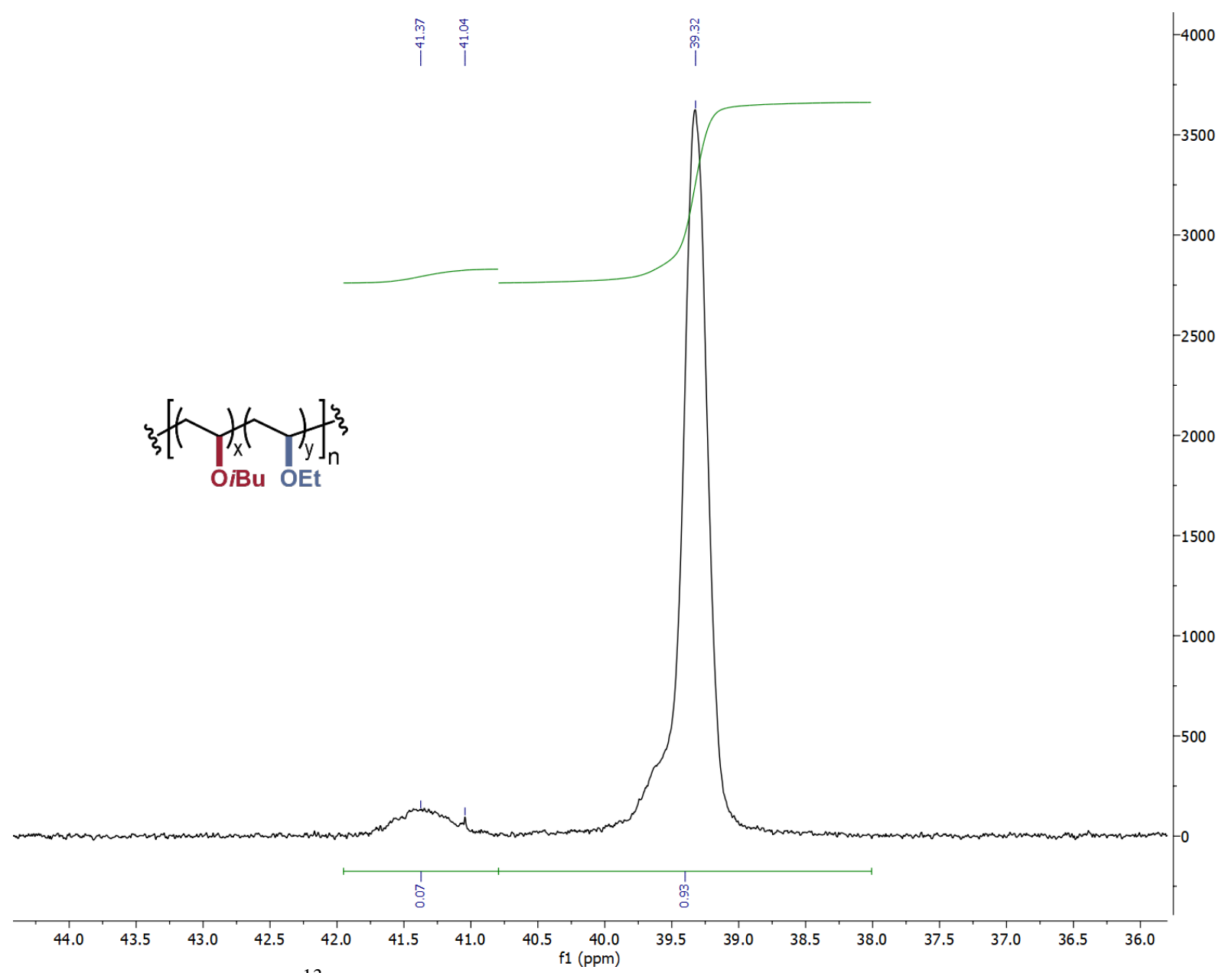

Figure S9. Expanded ${ }^{13} \mathrm{C}$ NMR spectrum of a representative sample of isotactic poly(iBVE-coEVE) showing relative integrations amounting to $93 \%$ meso diads. In this example, $F_{\mathrm{Et}}=0.52$ $\left(\mathrm{CDCl}_{3}\right)$. 


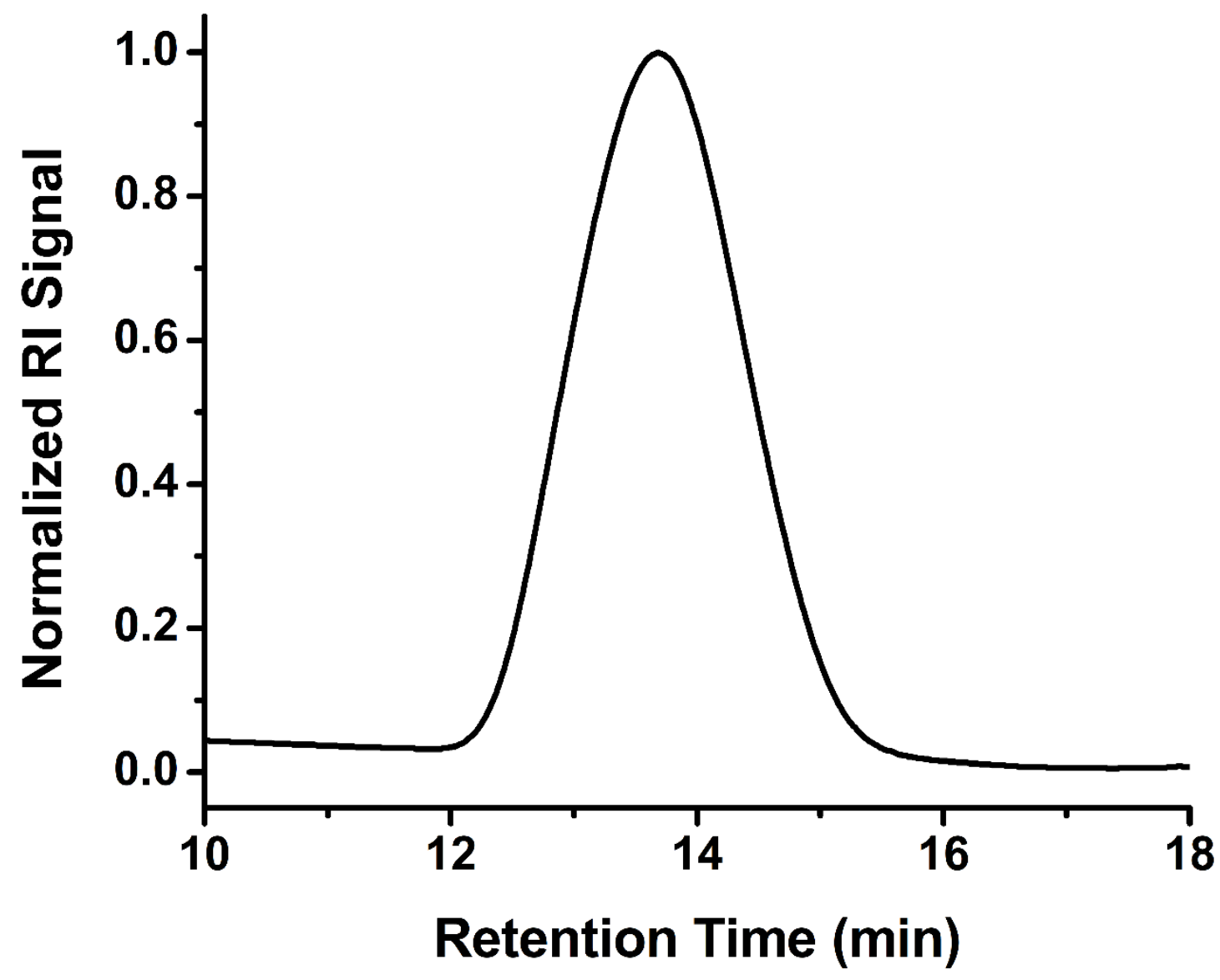

Figure S10. Representative GPC RI trace of isotactic poly(iBVE-co-EVE). In this example, $F_{\mathrm{Et}}=$ 0.09 . 


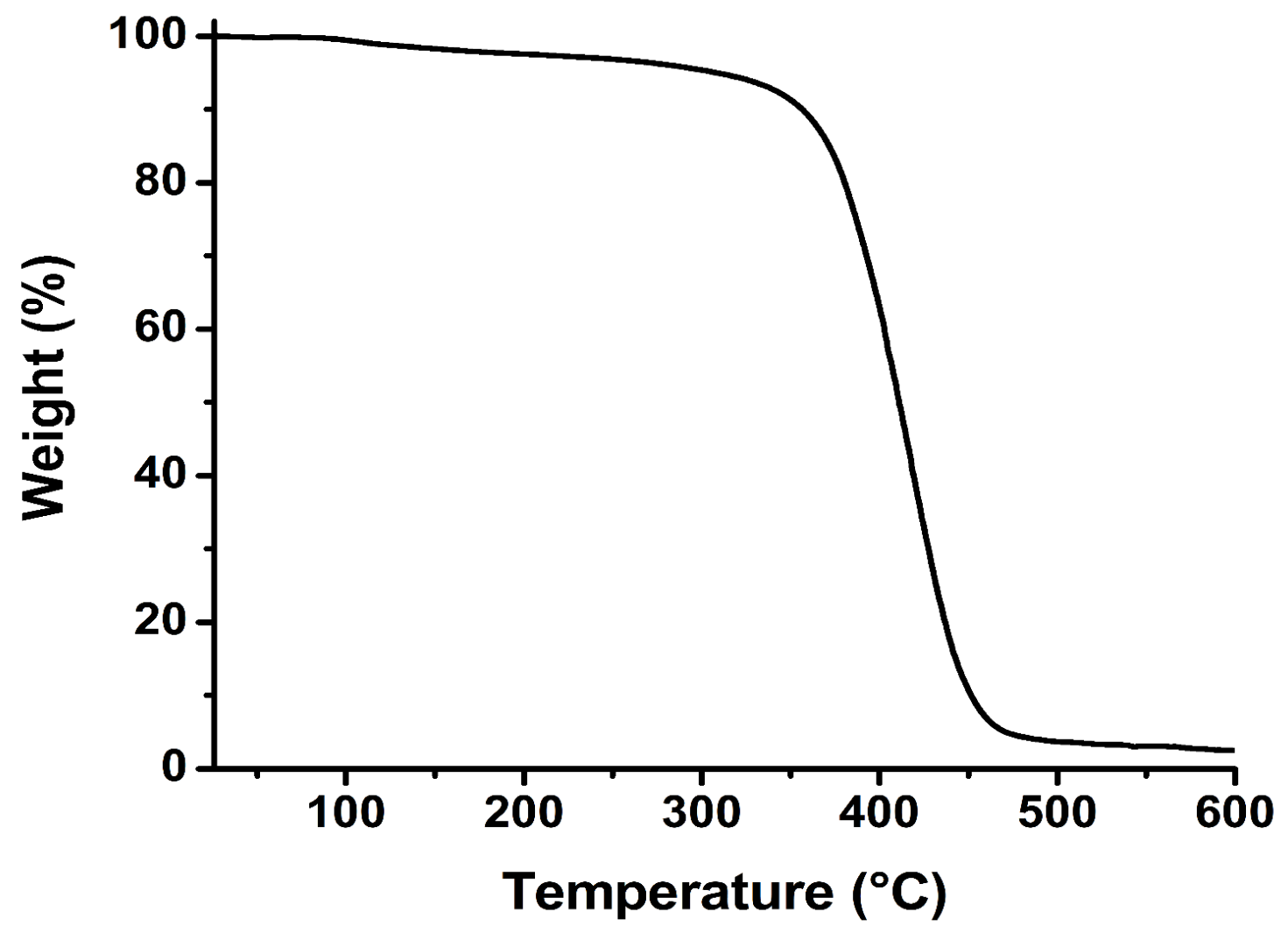

Figure S11. Representative TGA curve for isotactic poly(iBVE-co-EVE). In this example, $F_{\mathrm{Et}}=$ 0.52 . 


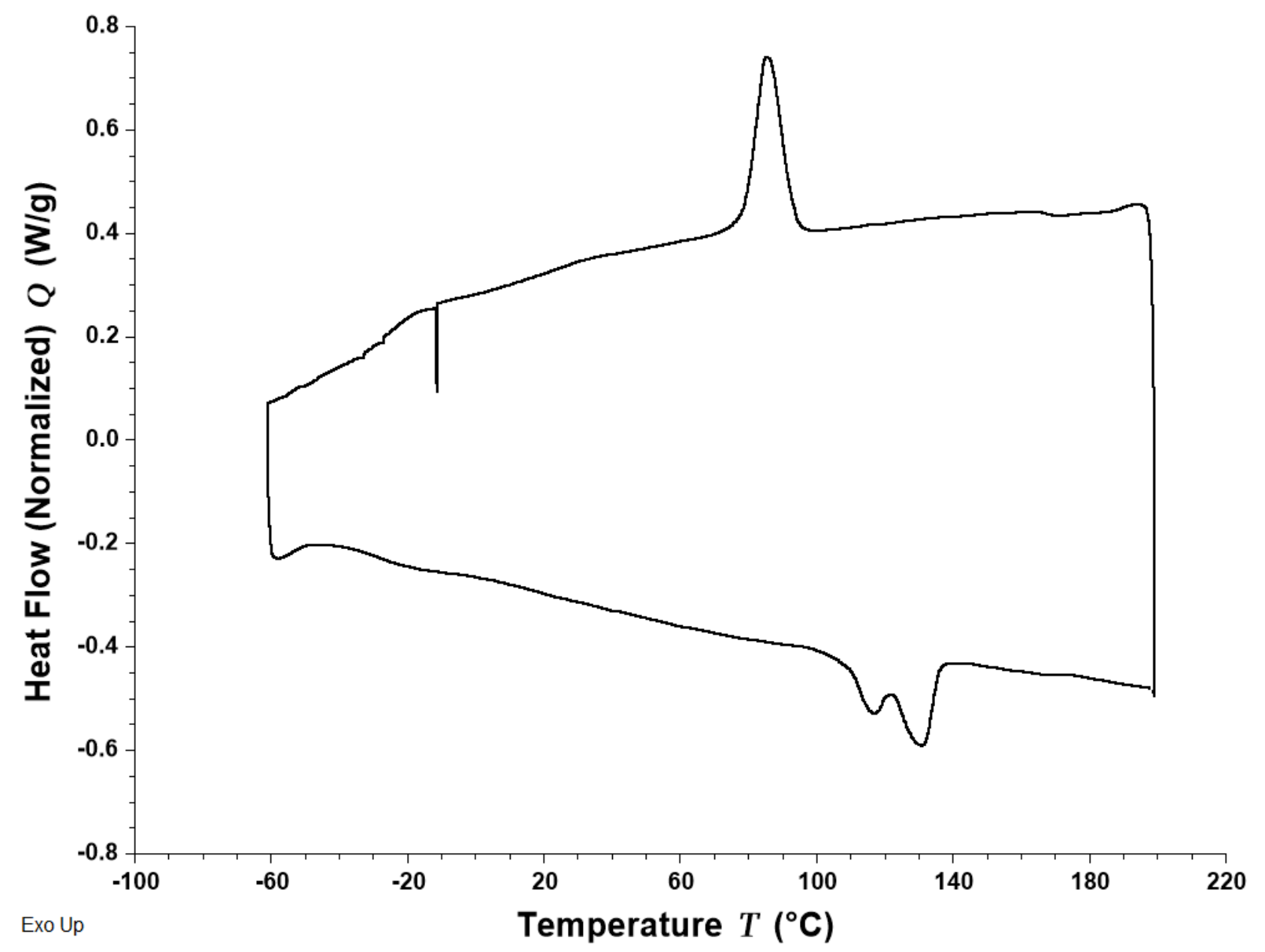

Figure S12. Representative DSC curves for isotactic poly(iBVE-co-EVE). In this example, $F_{\mathrm{Et}}=$ 0.09 . 


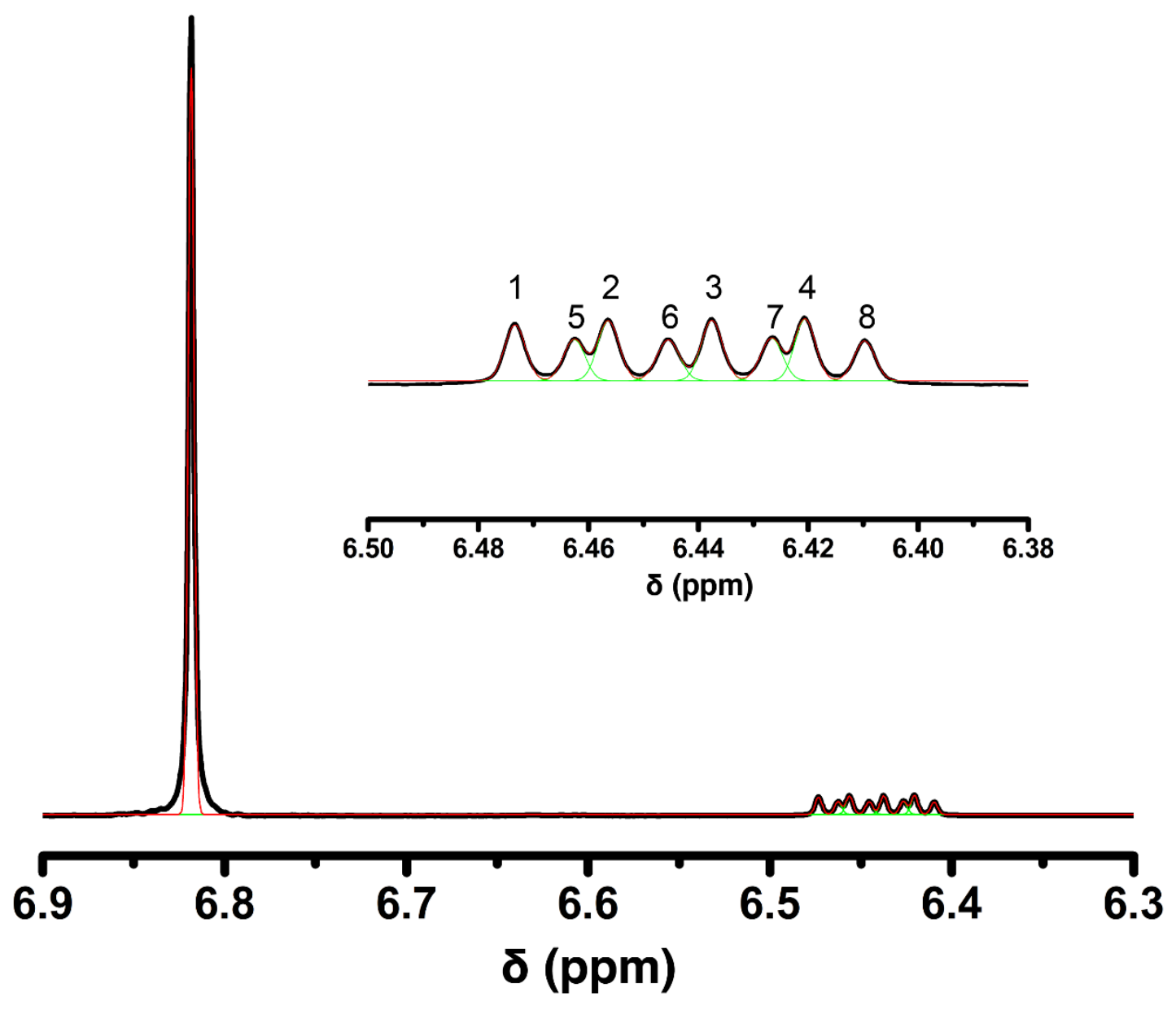

Figure S13. Representative example of peak deconvolution using OriginPro 8 to determine the relative consumption of individual vinyl ether monomers. The aryl resonance of the 1,4dimethoxybenzene internal standard can be seen at $\delta 6.8 \mathrm{ppm}$, while the overlapped vinyl resonances for iBVE and $\mathrm{nBVE}$ are between $\delta 6.40-6.48 \mathrm{ppm}$ (expanded in inset). This example represents a copolymerization of $\mathrm{nBVE}$ with iBVE with $f_{\mathrm{Bu}}=0.50$ that was quenched at $20 \min (t$ $=1200 \mathrm{~s}$ ). Fitted peaks (green) and peak sum (red) are shown overlaid on the original spectrum (black). 


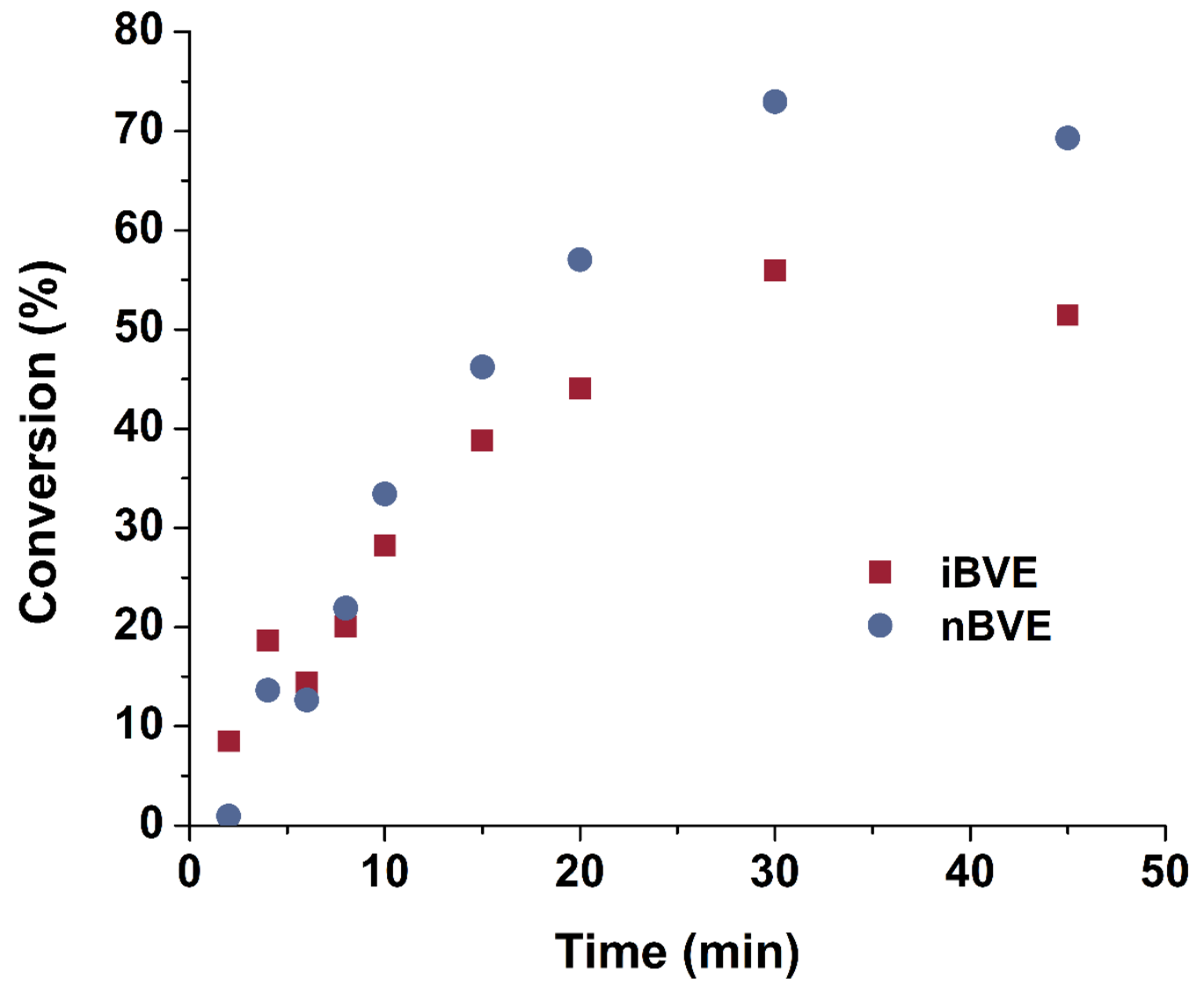

Figure S14. Plot of $\%$ conversion versus time of the copolymerization $\left(f_{\mathrm{Bu}}=0.50\right)$ of iBVE and nBVE. Conversions of iBVE ( $\bullet$ ) and nBVE ( $\bullet$ ) monitored independently by ${ }^{1} \mathrm{H}$ NMR $\left(\mathrm{CDCl}_{3}\right)$. $[\mathrm{iBVE}]_{0}=0.19 \mathrm{M} .[\mathrm{nBVE}]_{0}=0.19 \mathrm{M}$ 

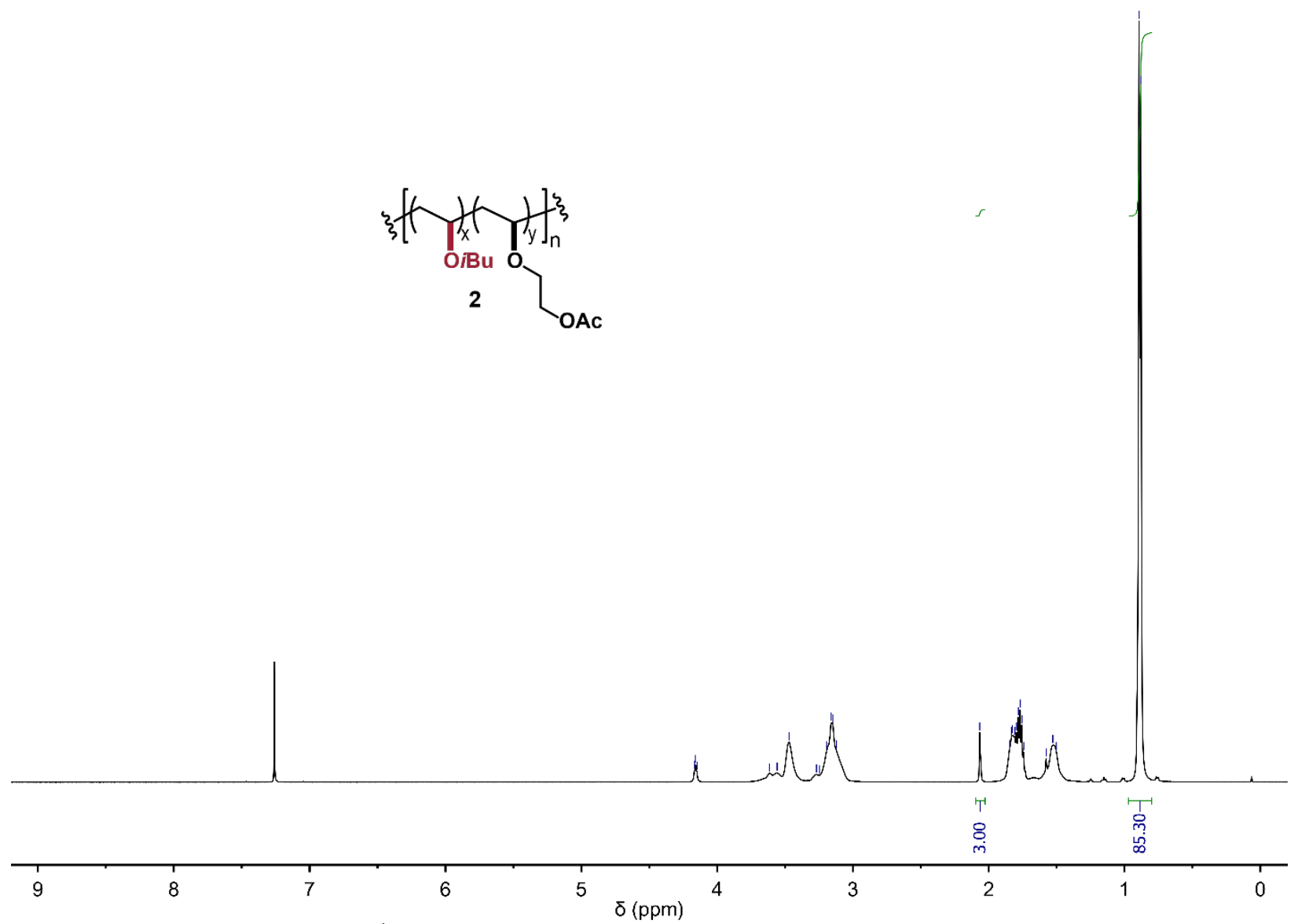

Figure S15. Representative ${ }^{1} \mathrm{H}$ NMR spectrum of isotactic poly(iBVE-co-AcVE). In this example, $F_{\mathrm{Ac}}=0.07\left(\mathrm{CDCl}_{3}\right)$. 


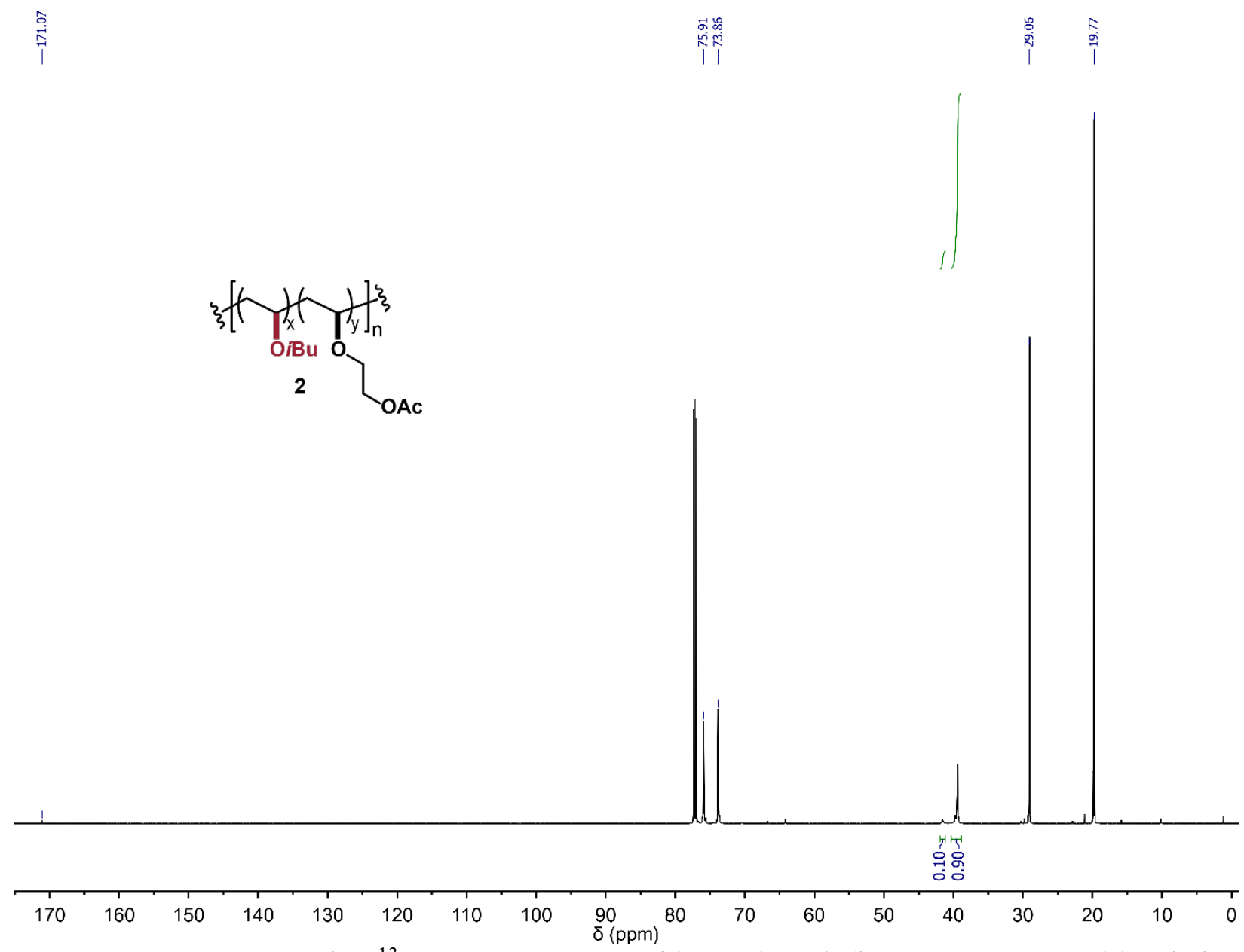

Figure S16. Representative ${ }^{13} \mathrm{C}$ NMR spectrum of isotactic poly(iBVE-co-AcVE) with relative integrations amounting to $90 \%$ meso diads. In this example, $F_{\mathrm{Ac}}=0.02\left(\mathrm{CDCl}_{3}\right)$. 

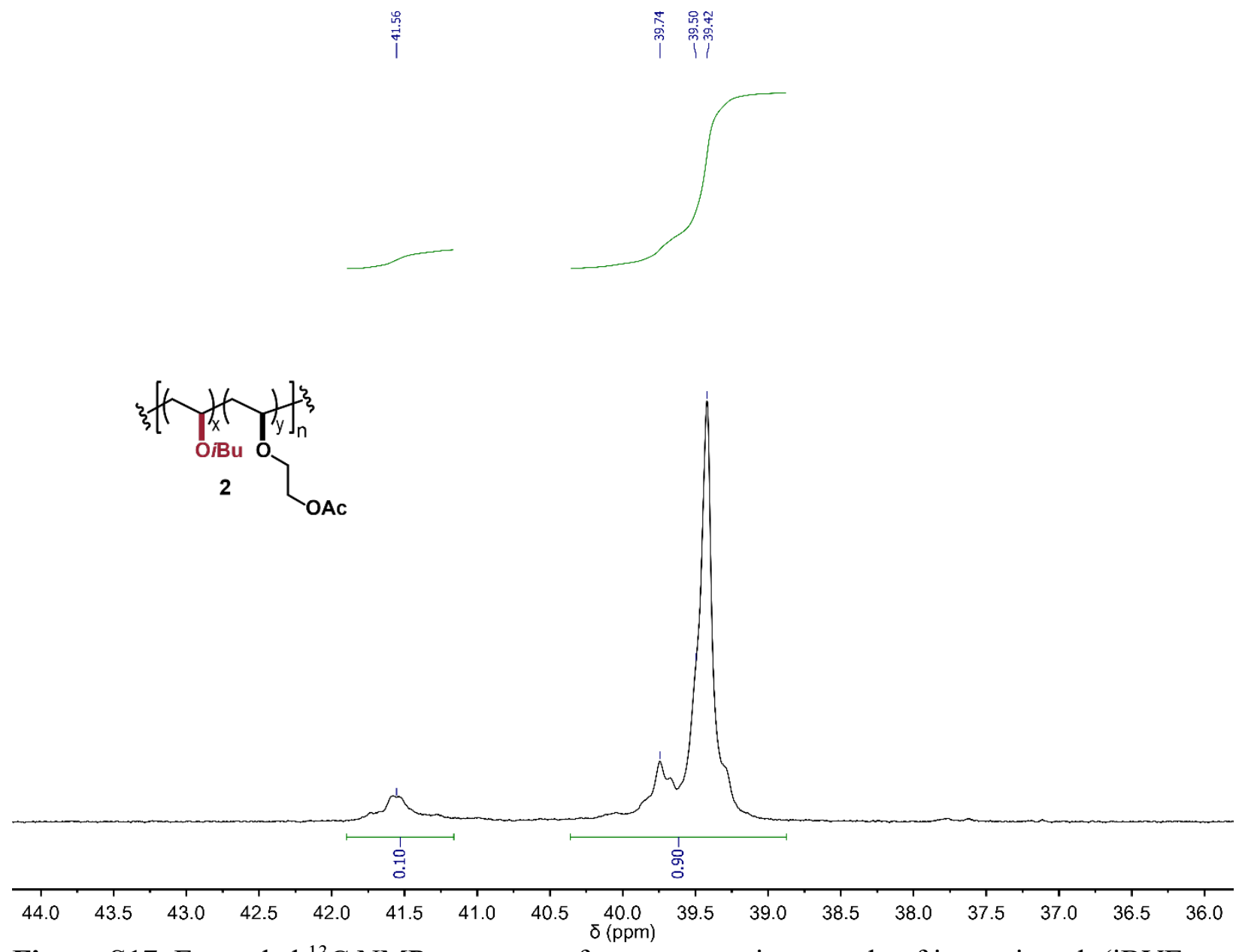

Figure S17. Expanded ${ }^{13} \mathrm{C}$ NMR spectrum of a representative sample of isotactic poly(iBVE-coAcVE) showing relative integrations amounting to $90 \%$ meso diads. In this example, $F_{\mathrm{Ac}}=0.02$ $\left(\mathrm{CDCl}_{3}\right)$. 


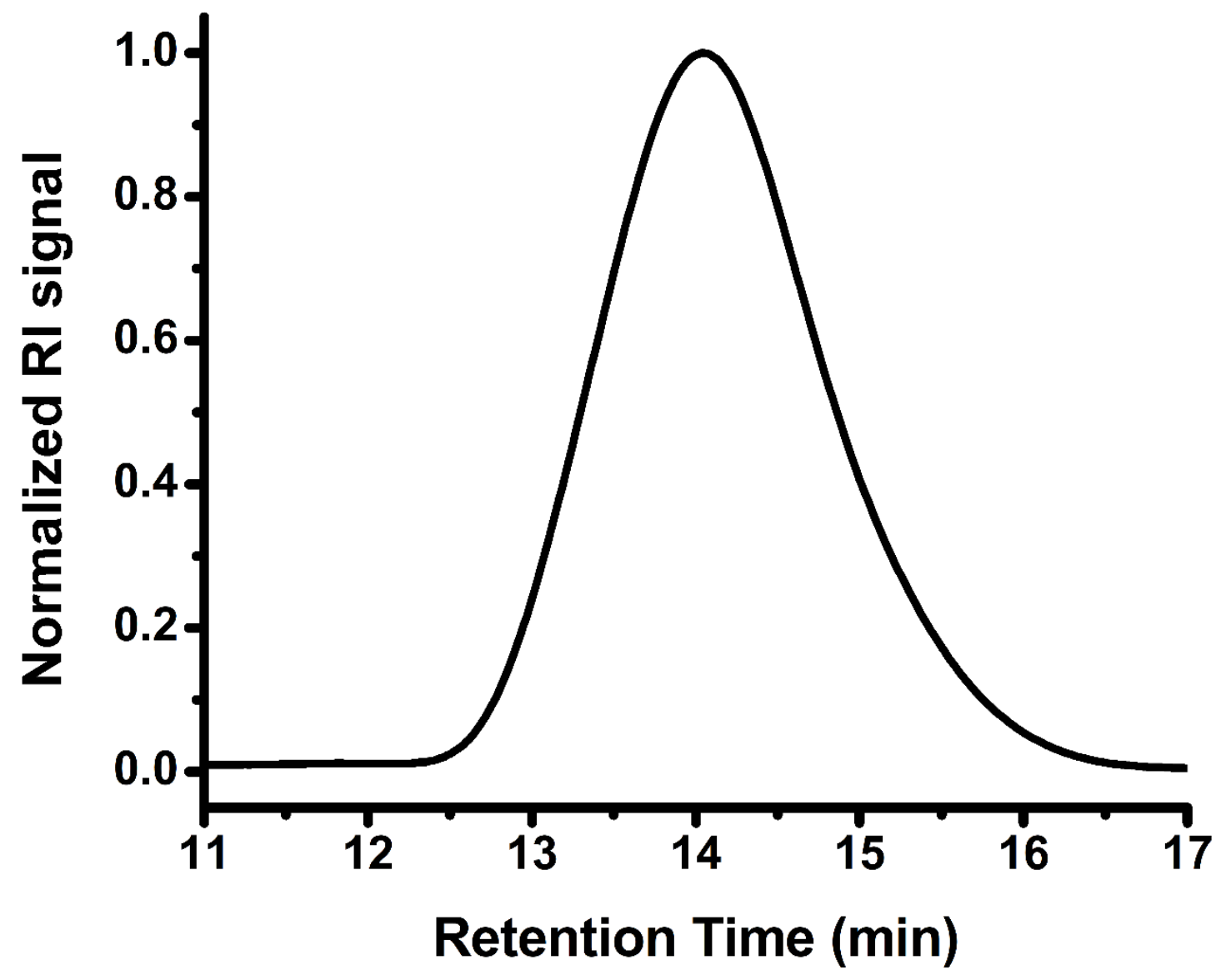

Figure S18. Representative GPC RI trace of isotactic poly(iBVE-co-AcVE). In this example, $F_{\text {Ac }}$ $=0.07$. 


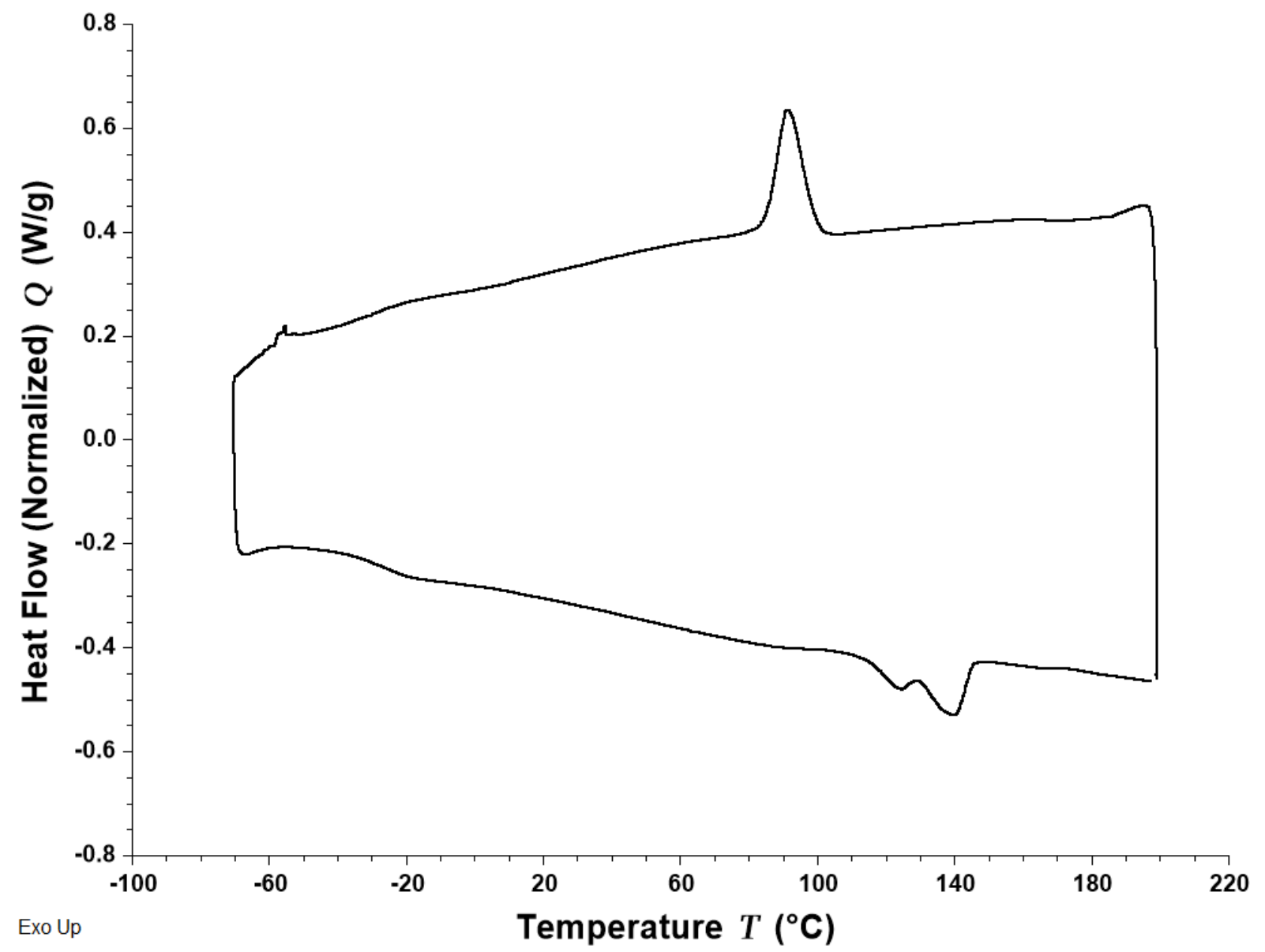

Figure S19. Representative DSC curves for isotactic poly(iBVE-co-AcVE). In this example, $F_{\text {Ac }}$ $=0.07$. 

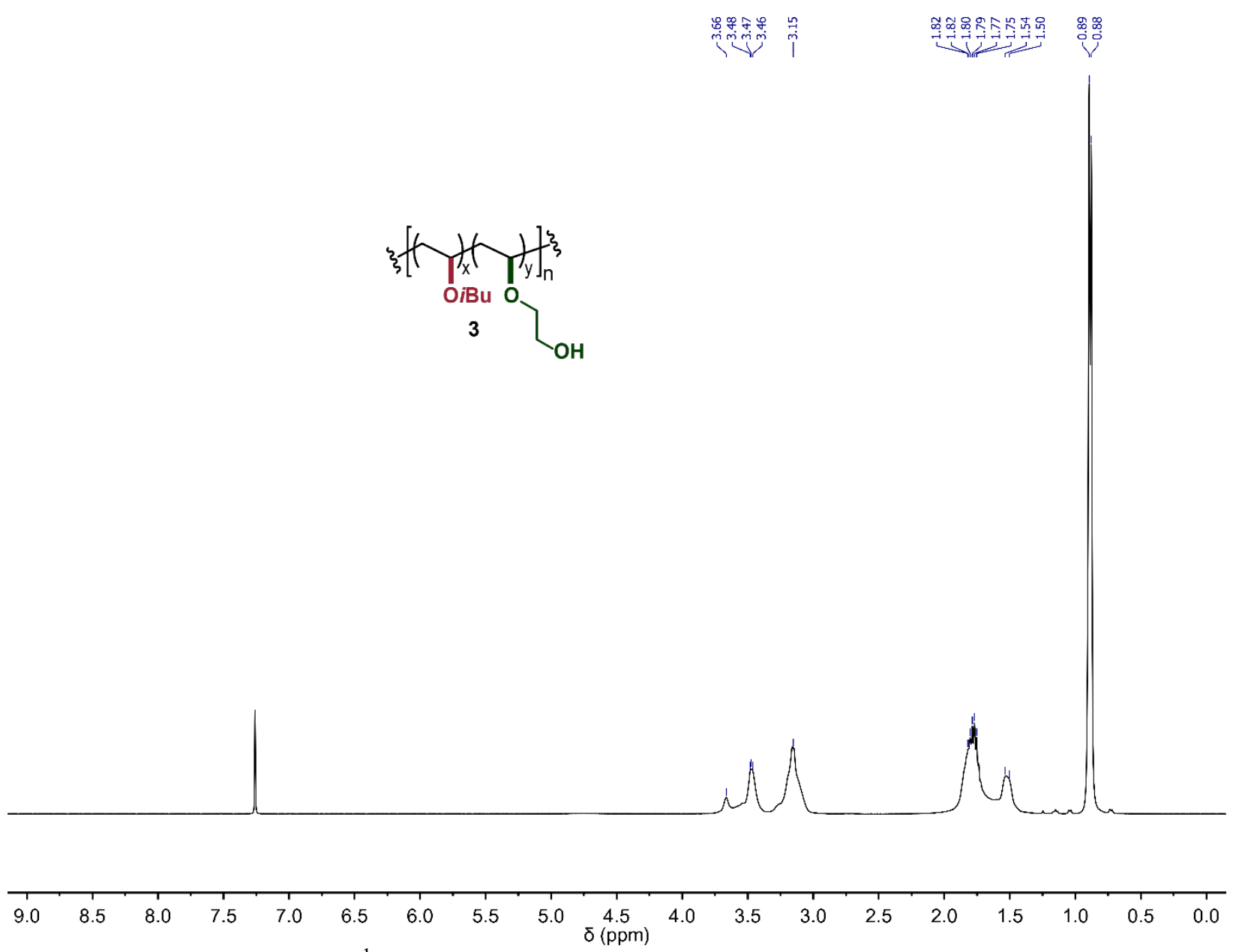

Figure S20. Representative ${ }^{1} \mathrm{H}$ NMR spectrum of isotactic copolymer $3\left(\mathrm{CDCl}_{3}\right)$. 


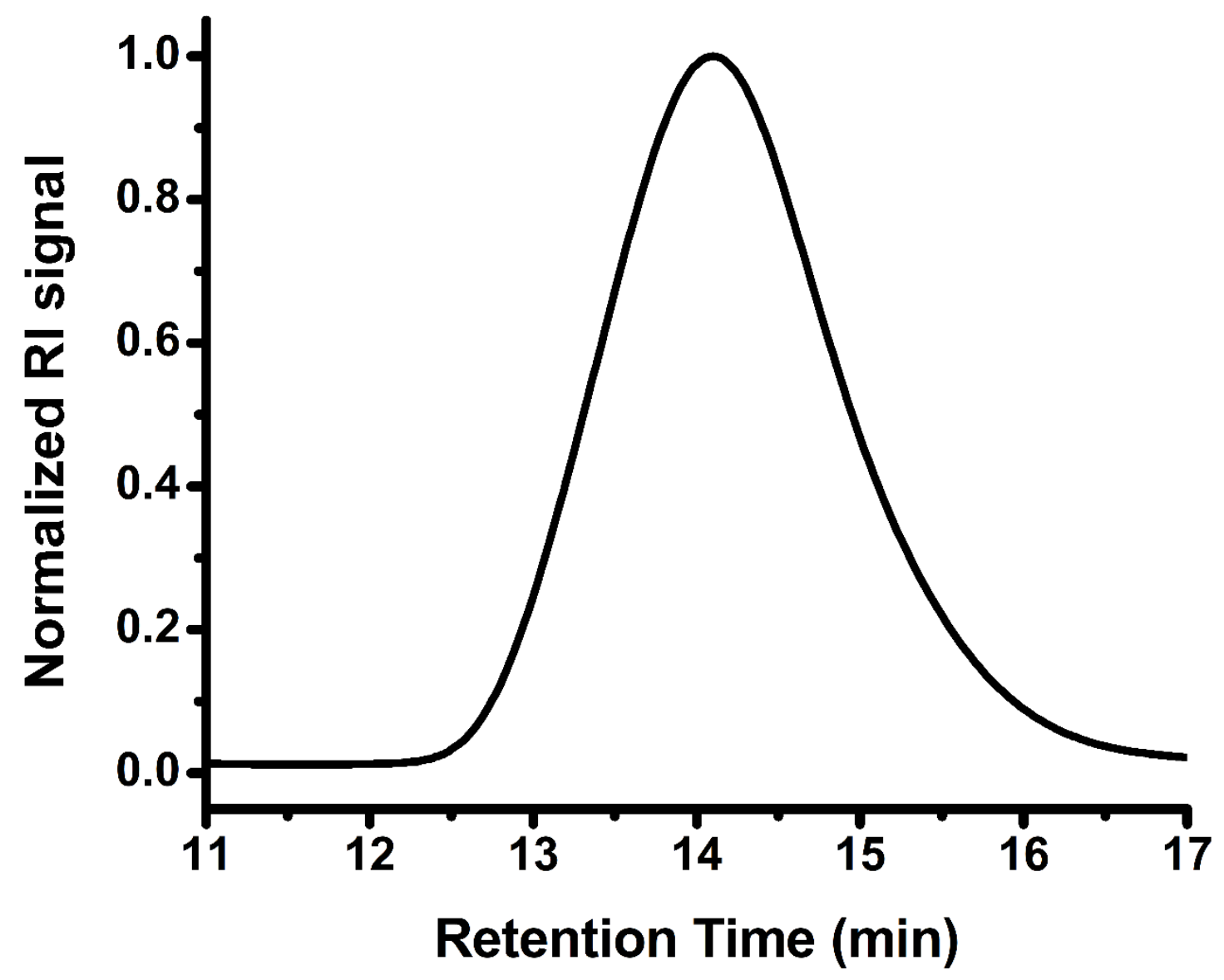

Figure S21. Representative GPC RI trace of isotactic copolymer 3. 


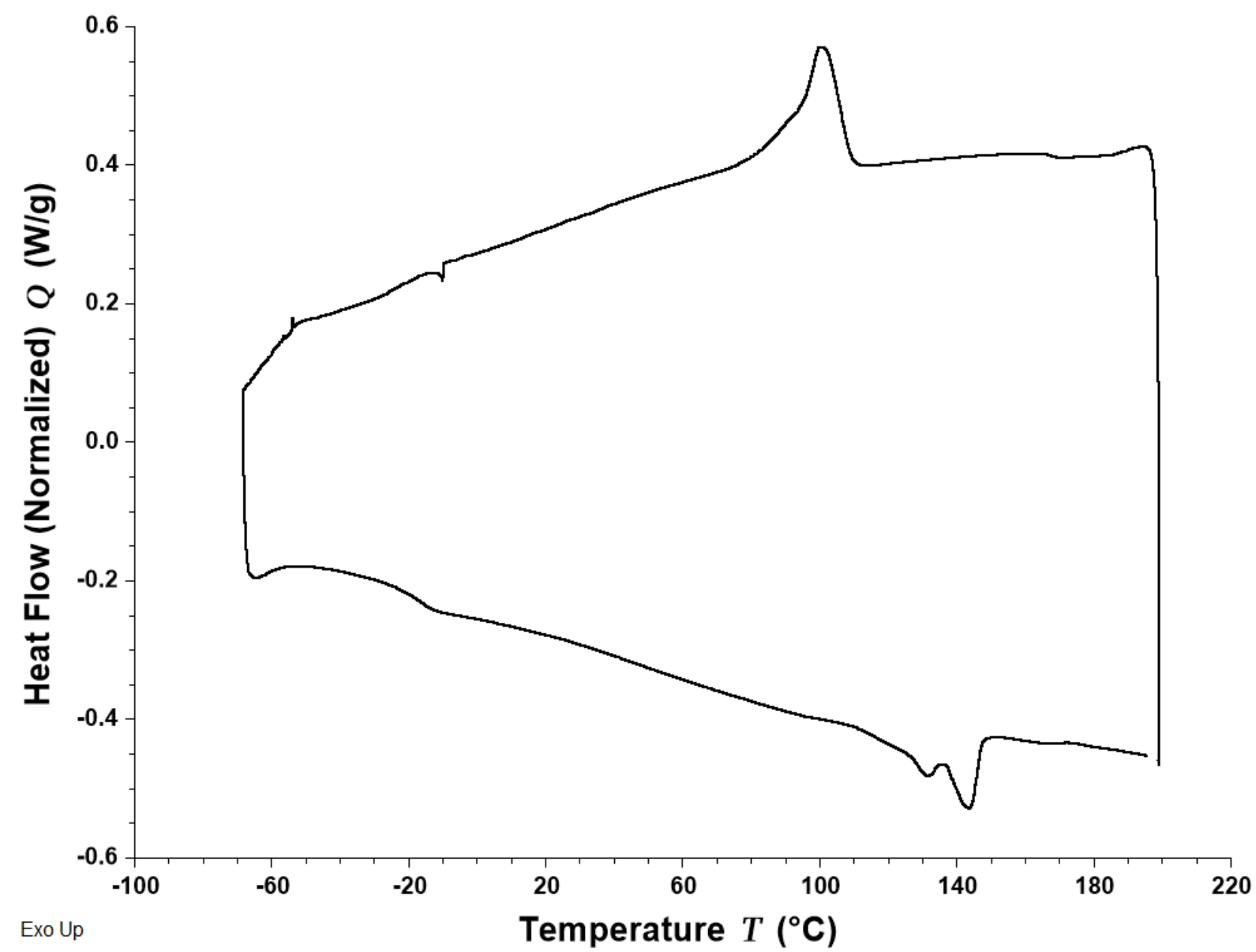

Figure S22. Representative DSC curves for isotactic copolymer 3. 

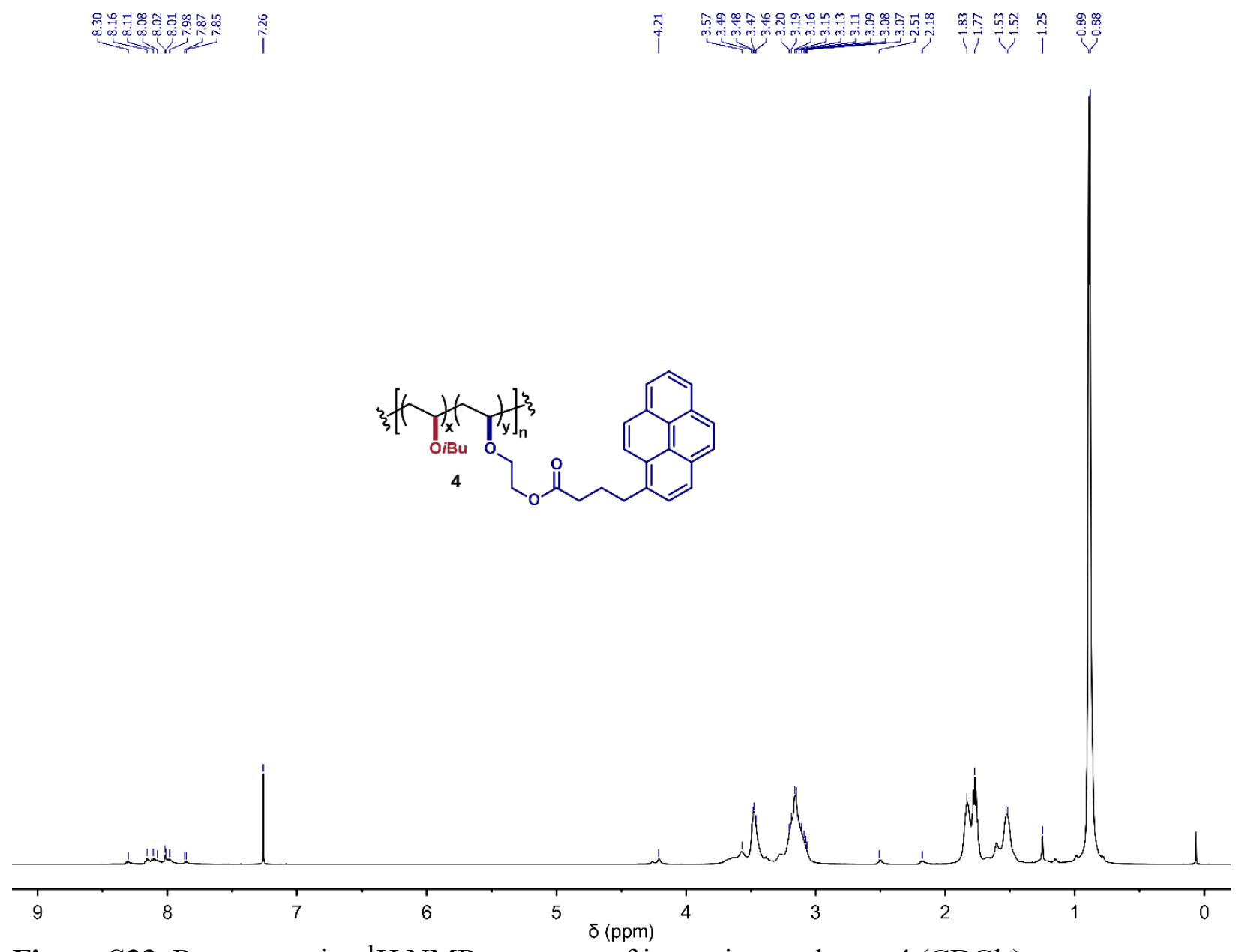

Figure S23. Representative ${ }^{1} \mathrm{H}$ NMR spectrum of isotactic copolymer $4\left(\mathrm{CDCl}_{3}\right)$. 


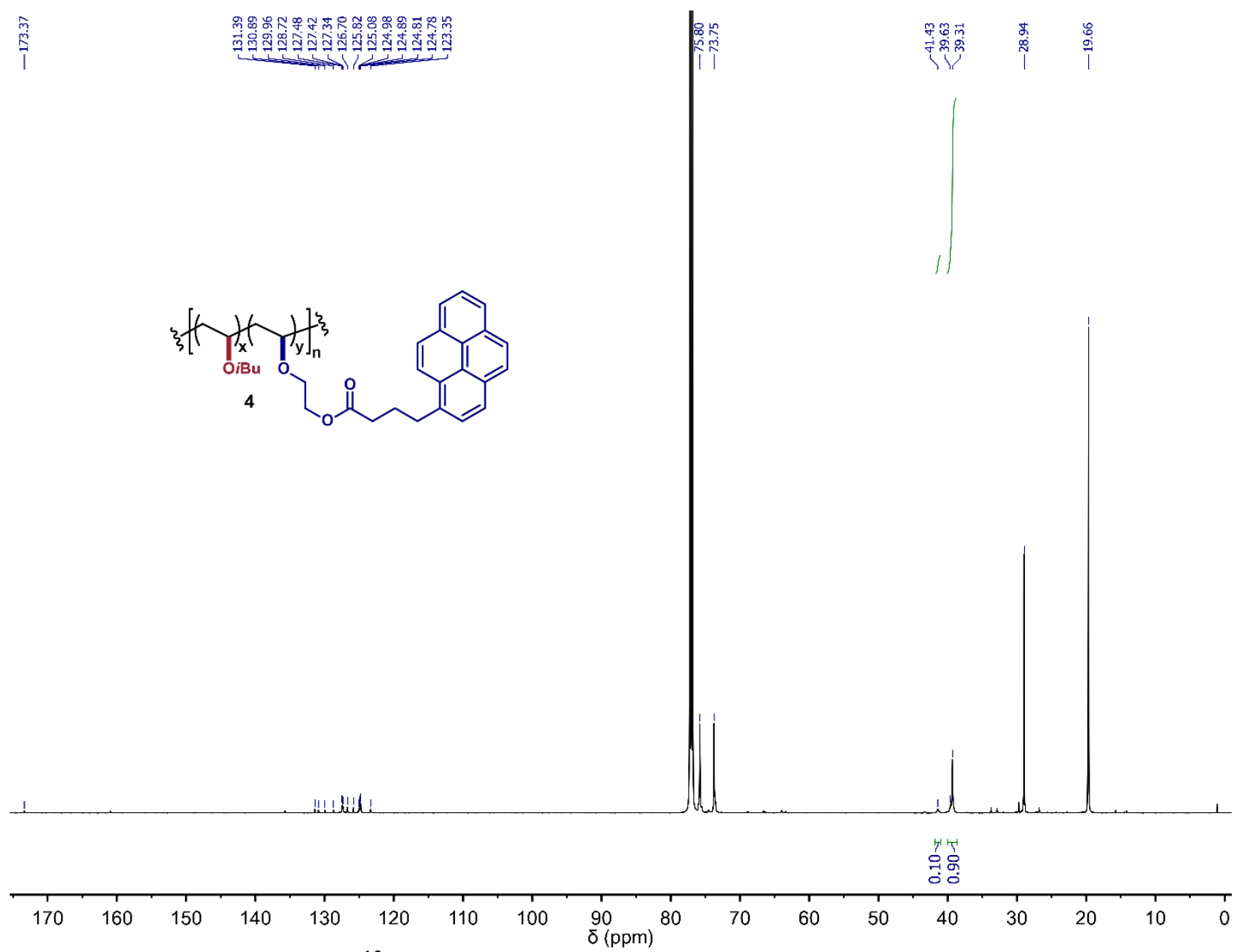

Figure S24. Representative ${ }^{13} \mathrm{C}$ NMR spectrum of isotactic copolymer 4 with relative integrations amounting to $90 \%$ meso diads $\left(\mathrm{CDCl}_{3}\right)$. 


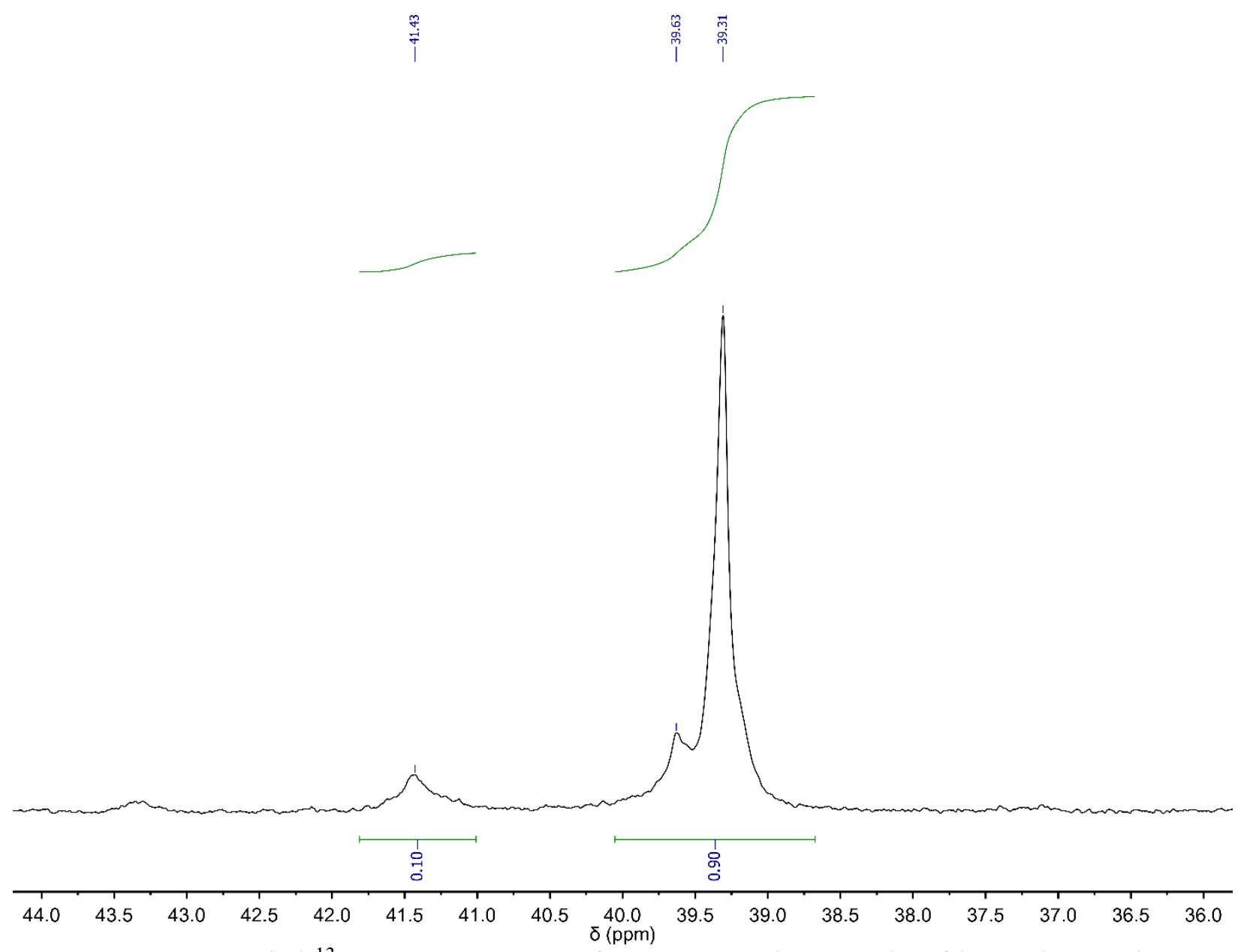

Figure S25. Expanded ${ }^{13} \mathrm{C}$ NMR spectrum of a representative sample of isotactic copolymer 4 showing relative integrations amounting to $90 \%$ meso diads $\left(\mathrm{CDCl}_{3}\right)$. 


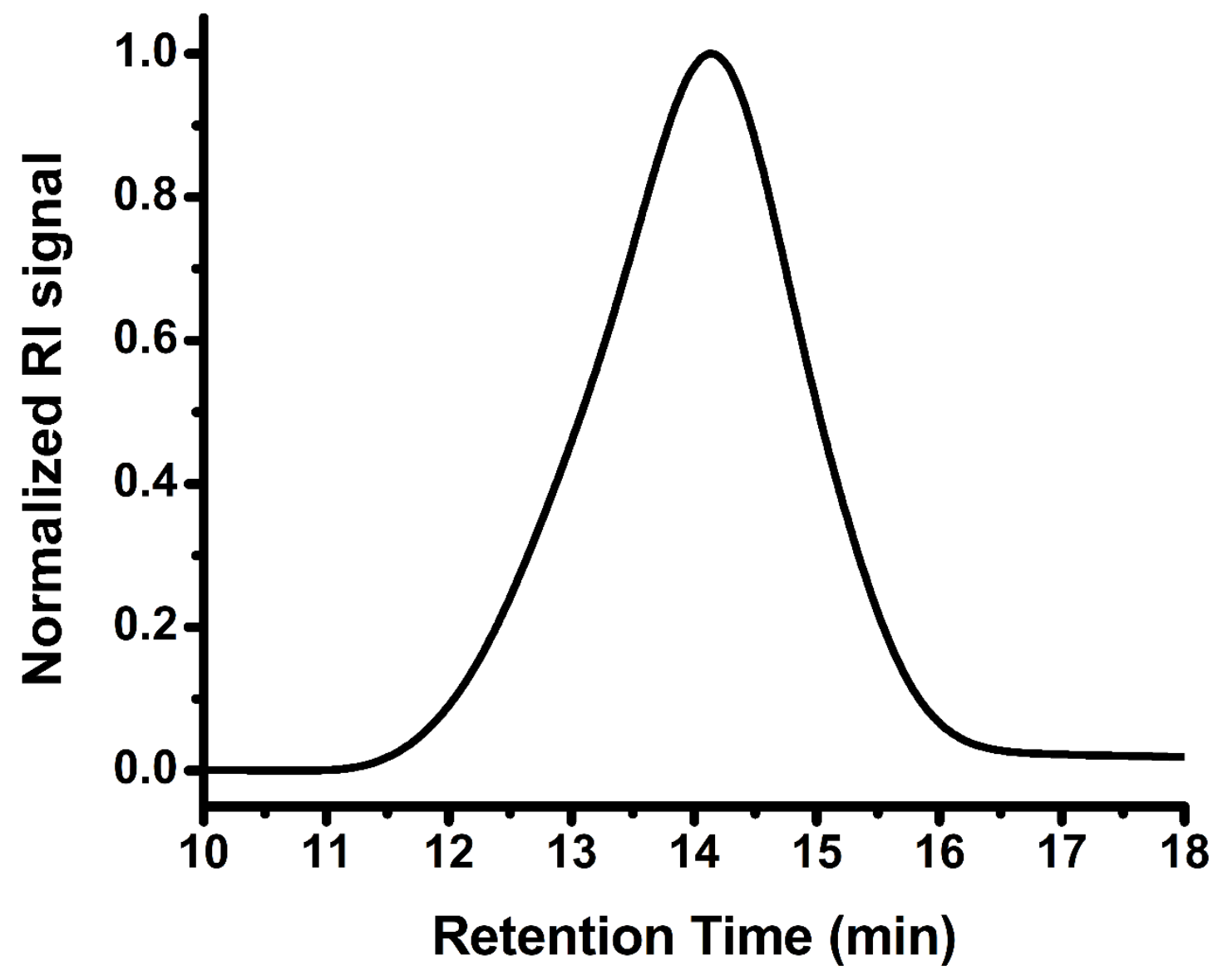

Figure S26. Representative GPC RI trace of isotactic copolymer 4. 


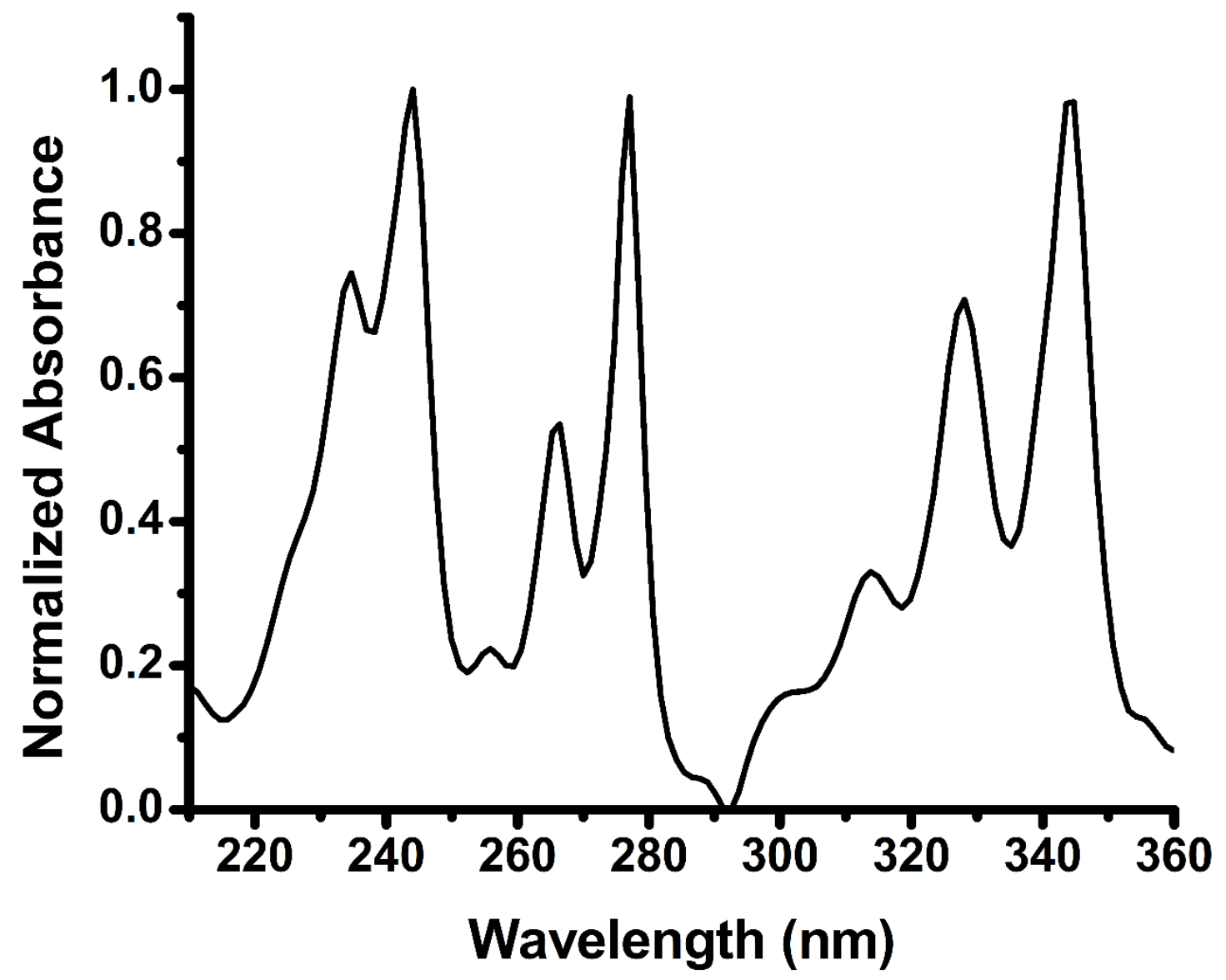

Figure S27. Representative GPC PDA trace at $344 \mathrm{~nm}$ of isotactic copolymer 4, confirming the presence of pyrene-derived repeat units. 


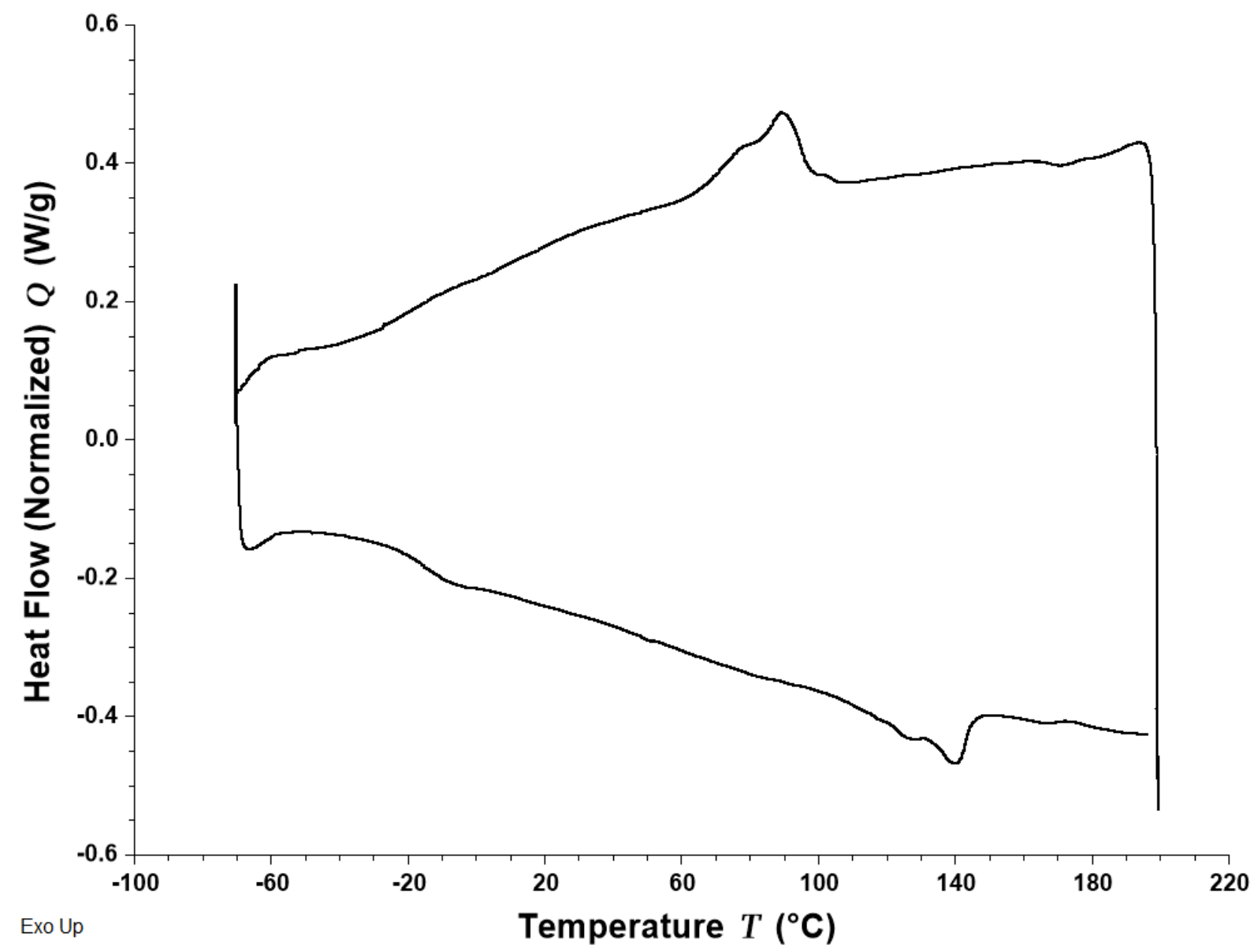

Figure S28. Representative DSC curves for isotactic copolymer 4. 


\section{References}

(1) Akiyama, T.; Morita, H.; Itoh, J.; Fuchibe, K. Chiral Brønsted Acid Catalyzed Enantioselective Hydrophosphonylation of Imines: Asymmetric Synthesis of $\alpha$-Amino Phosphonates. Org. Lett. 2005, 7 (13), 2583-2585. https://doi.org/10.1021/OL050695E.

(2) Manxzer, L. E.; Deaton, J.; Sharp, P.; Schrock, R. R. Tetrahydrofuran Complexes of Selected Early Transition Metals. In Inorganic Syntheses; John P. Fackler Jr., Ed.; John Wiley \& Sons, Ltd, 1982; pp 135-140. https://doi.org/10.1002/9780470132524.ch31.

(3) Lai, T.-L.; Pop, F.; Melan, C.; Canevet, D.; Sallé, M.; Avarvari, N. Triggering Gel Formation and Luminescence through Donor-Acceptor Interactions in a C3-Symmetric Tris(Pyrene) System. Chem. - A Eur. J. 2016, 22 (17), 5839-5843. https://doi.org/10.1002/chem.201600072.

(4) OriginPro, Version 8. OriginLab Corporation, Northampton, MA, USA. 